\title{
Article
}

\section{The nuclear activity and central structure of the elliptical galaxy NGC 5322}

Dullo, Bililign T, Knapen, Johan H, Williams, David R A, Beswick, Robert J, Bendo, George, Baldi, Ranieri D, Argo, Megan K., McHardy, Ian M, Muxlow, Tom and Westcott, J

Available at http://clok.uclan.ac.uk/21454/

Dullo, Bililign T, Knapen, Johan H, Williams, David R A, Beswick, Robert J, Bendo, George, Baldi, Ranieri D, Argo, Megan K. ORCID: 0000-0003-35940214, McHardy, lan M, Muxlow, Tom et al (2018) The nuclear activity and central structure of the elliptical galaxy NGC 5322. Monthly Notices of the Royal Astronomical Society . ISSN 0035-8711

It is advisable to refer to the publisher's version if you intend to cite from the work. http://dx.doi.org/10.1093/mnras/sty069

For more information about UCLan's research in this area go to http://www.uclan.ac.uk/researchgroups/ and search for <name of research Group>.

For information about Research generally at UCLan please go to http://www.uclan.ac.uk/research/

All outputs in CLoK are protected by Intellectual Property Rights law, including Copyright law. Copyright, IPR and Moral Rights for the works on this site are retained by the individual authors and/or other copyright owners. Terms and conditions for use of this material are defined in the policies page. 


\title{
The nuclear activity and central structure of the elliptical galaxy NGC 5322
}

\author{
Bililign T. Dullo, ${ }^{1,2,3 \star}$, Johan H. Knapen ${ }^{2,3}$, David R. A. Williams ${ }^{4}$, Robert J. \\ Beswick $^{5}$, George Bendo ${ }^{5}$, Ranieri D. Baldi ${ }^{4}$, Megan Argo ${ }^{5,6}$, Ian M. McHardy ${ }^{4}$, \\ Tom Muxlow ${ }^{5}$, J. Westcott ${ }^{7}$ \\ ${ }^{1}$ Departamento de Astrofísica y Ciencias de la Atmósfera, Universidad Complutense de Madrid, E-28040 Madrid, Spain \\ ${ }^{2}$ Instituto de Astrofísica de Canarias, Vía Láctea S/N, E-38205, La Laguna, Tenerife, Spain \\ ${ }^{3}$ Departamento de Astrofísica, Universidad de La Laguna, E-38206, La Laguna, Tenerife, Spain \\ ${ }^{4}$ School of Physics and Astronomy, University of Southampton, Southampton, SO17 1BJ, UK \\ ${ }^{5}$ Jodrell Bank Centre for Astrophysics, School of Physics and Astronomy, The University of Manchester, Alan Turing Building, Oxford Road, \\ Manchester, M13 9PL, UK \\ ${ }^{6}$ Jeremiah Horrocks Institute, University of Central Lancashire, Preston PR1 2HE, UK \\ ${ }^{7}$ Centre for Astrophysics Research, University of Hertfordshire, College Lane, Hatfield, AL10 9AB, UK
}

9 January 2018

\begin{abstract}
We have analysed a new high-resolution e-MERLIN $1.5 \mathrm{GHz}$ radio continuum map together with HST and SDSS imaging of NGC 5322, an elliptical galaxy hosting radio jets, aiming to understand the galaxy's central structure and its connection to the nuclear activity. We decomposed the composite HST + SDSS surface brightness profile of the galaxy into an inner stellar disc, a spheroid, and an outer stellar halo. Past works showed that this embedded disc counter-rotates rapidly with respect to the spheroid. The $H S T$ images reveal an edge-on nuclear dust disc across the centre, aligned along the major-axis of the galaxy and nearly perpendicular to the radio jets. After careful masking of this dust disc, we find a central stellar mass deficit $M_{\text {def }}$ in the spheroid, scoured by SMBH binaries with final mass $M_{\mathrm{BH}}$ such that $M_{\mathrm{def}} / M_{\mathrm{BH}} \sim 1.3-3.4$. We propose a three-phase formation scenario for NGC 5322 where a few $(2-7)$ 'dry' major mergers involving SMBHs built the spheroid with a depleted core. The cannibalism of a gas-rich satellite subsequently creates the faint counter-rotating disc and funnels gaseous material directly onto the AGN, powering the radio core with a brightness temperature of $T_{\mathrm{B}, \text { core }} \sim 4.5 \times 10^{7} \mathrm{~K}$ and the low-power radio jets $\left(P_{\text {jets }} \sim 7.04 \times 10^{20} \mathrm{~W} \mathrm{~Hz}^{-1}\right)$ which extend $\sim 1.6 \mathrm{kpc}$. The outer halo can later grow via minor mergers and the accretion of tidal debris. The low-luminosity AGN/jet-driven feedback may have quenched the late-time nuclear star formation promptly, which could otherwise have replenished the depleted core.
\end{abstract}

Key words: galaxies: elliptical and lenticular, $\mathrm{cD}$ - galaxies: nuclei — galaxies: photometry — galaxies: structure — radio continuum: galaxies

\section{INTRODUCTION}

Elliptical galaxies are thought to have formed hierarchically via mergers of smaller systems and accretion events (e.g., White \& Rees 1978; Cole et al. 1994; Khochfar \& Burkert 2001; Springel et al. 2005; Naab, Khochfar, \& Burkert 2006; Hopkins et al. 2009; Mundy et al. 2017). Merger-driven inflow of gas was invoked to produce nuclear starbursts or fuel the central black hole. The latter may drive powerful relativistic jets that can heat the surrounding gas, and as such impact on the central stellar structures in elliptical galaxies (e.g., Carlberg 1986; Hernquist 1992, 1993; Mihos \& Hernquist 1994; Bahcall et al. 1995; Surace et al. 2001; Croton et al. 2006; Werner et al. 2014; Somerville \& Davé 2015). Observations reveal that elliptical galaxies

* bdullo@ucm.es 
possess distinct structures on scales of a few kiloparsecs down to tens of parsecs such as depleted cores, nuclear star clusters, small-scale stellar discs, nuclear dust discs/lanes, and AGN (e.g., Crane et al. 1993; Kormendy et al. 1994; Jaffe et al. 1994; Ferrarese et al. 1994; Lauer et al. 1995; Carollo et al. 1997, Faber et al. 1997; Knapen et al. 2000; Rest et al. 2001; Trujillo et al. 2004; Lauer et al. 2005, 2007; Côté et al. 2006; Ferrarese et al. 2006; Kormendy et al. 2009; Dullo \& Graham 2012, 2014; Turner et al. 2012; Savorgnan \& Graham 2016; Dullo, Graham, \& Knapen 2017). However, the interplay between such central structures and the nuclear activity of the galaxies remain largely unclear.

Luminous elliptical galaxies brighter than $M_{\mathrm{B}} \sim-20.5 \pm 0.05$ mag contain partially-depleted cores, with sizes typically $20-500 \mathrm{pc}$, that are thought to be scoured by binary supermassive black holes formed in gas-poor ('dry') major mergers (e.g., Begelman et al. 1980; Ebisuzaki et al. 1991; Faber et al. 1997; Merritt 2006). The coexistence of such cores and rapidly rotating nuclear stellar and dust discs in galaxies is of interest since it may lead us to understand how such galaxies can acquire and transfer gaseous material inward to build the nuclear stellar/dust discs, fuel the central AGN, but not refill the depleted core with young stars. The radio elliptical (E3-4) galaxy NGC 5322 (RC3, de Vaucouleurs et al. 1991), with a depleted core, i.e., a central stellar mass deficit (e.g., Carollo et al. 1997; Richings et al. 2011; Krajnović et al. 2013a), nuclear stellar disc and LINER-type nuclear emission (Baldi \& Capetti 2009), is a promising candidate which allows detailed, multi-wavelength analysis of the central structure, AGN activities and jet structures.

The Legacy e-MERLIN Multi-band Imaging of Nearby Galaxies (LeMMINGs; Beswick et al. 2015) survey is a highresolution radio continuum survey designed, in part, to study such galaxies by utilising synergies from a large sample of high-resolution, multi-wavelength data, including from HST: optical plus IR, Spitzer: IR, Herschel: IR, Chandra: X-ray and GALEX: UV. The project is composed of two complementary radio surveys: moderately deep snapshots of a large sample of 280 nearby $(3-100 \mathrm{Mpc}$ ) galaxies over a large luminosity, size and morphological type range, selected from the Palomar Bright Galaxies Survey (Ho et al. 1997), and a deep survey of 6/280 representative galaxies. These radio surveys coupled with the ancillary data will probe the central structures, AGN/jet structure and star formation rates in galaxies (see Westcott et al. 2017 and Williams et al. 2017). NGC 5322 is among the first galaxies observed with e-MERLIN at 1.5 GHz as part of the moderately deep LeMMINGs survey.

Isophotal analysis of ground-based optical images by Bender (1988) showed that the isophote shapes in NGC 5322 change from discy isophotes at $R \lesssim 8^{\prime \prime}$, due to the galaxy's small-scale, edge-on stellar disc, to boxy isophotes at larger radii $\left(8^{\prime \prime} \lesssim R \lesssim 35^{\prime \prime}\right)$. Embedded discs are common in elliptical galaxies (e.g., Rest et al. 2001, references therein; Dullo \& Graham 2012; Graham, Ciambur, \& Savorgnan 2016). The Bender (1988) kinematic data for NGC 5322, out to 35", also revealed that the inner region $\left(R \lesssim 2.6^{\prime \prime}\right)$, where the embedded stellar disc contributes light significantly, is counter-rotating with respect to the rest of the galaxy (see also Bender \& Surma 1992, Rix \& White 1992 and Krajnović et al. 2011, their Fig C4). This counter-rotating core (KDC) exhibits enhanced $\mathrm{Mg}_{2}$ relative to the outer parts of the galaxy (Bender \& Surma 1992; Kobayashi \& Arimoto 1999). The $\mathrm{Mg}_{2}$ line index is thought to trace primarily metallicity. The stellar population analysis within $1 R_{\mathrm{e}}$ by Spolaor et al. (2010, and references therein) has revealed that the galaxy is very metal rich $([\mathrm{Z} / \mathrm{H}] \sim 0.45)$ with a super-solar $\alpha$-abundance ratio $([\alpha / \mathrm{Fe}] \sim 0.30)$ in the central region. Carollo et al. (1997) found that the $V-I$ colour of the KDC differs only slightly ( $\lesssim 0.02 \mathrm{mag}$ ) from that of the surrounding regions. These isophotal, kinematical and stellar population properties and the colour of NGC 5322 were attributed to the build-up of the galaxy through a major merger of two gas-rich galaxies (e.g., Schweizer et al. 1990; Hernquist \& Barnes 1991; Bender \& Surma 1992; Schweizer \& Seitzer 1992). However, the presence of a depleted core in NGC 5322 argues against such a gas-rich, major merger scenario in which the merger-driven gas dissipation and ensuing nuclear starburst are thought to produce coreless galaxies.

Reducing the Tonry et al. (2001) distance moduli by 0.06 mag (Blakeslee et al. 2002) yields a distance of $30.3 \mathrm{Mpc}$ for NGC 5322. The galaxy has a central velocity dispersion $\sigma \sim 229 \mathrm{~km} \mathrm{~s}^{-1}$ (HyperLeda ${ }^{1}$, Paturel et al. 2003). We assume that $\mathrm{H}_{0}=70 \mathrm{~km} \mathrm{~s}^{-1} \mathrm{Mpc}^{-1}$ and that 1 arcsec corresponds to $145 \mathrm{pc}$.

The aim of this work is to investigate the formation mechanisms for NGC 5322 using a multi-wavelength analysis of the galaxy's central structure and the connection to the nuclear activity. To achieve this, we use our new 1.5 GHz e-MERLIN observations plus HST and SDSS imaging. These data and the pertaining data reduction steps are discussed in Section 2. After carefully masking the galaxy's nuclear dust disc, for the first time, we decompose the 200" composite (HST + SDSS) light profile into an inner (exponential) stellar disc, a core-Sérsic spheroid and an outer exponential stellar halo (Section 3). Section 3 also details our radio, isophotal and light profile fitting analyses, along with a literature comparison. Sections 4.1 and 4.2 provide our measurements of the central stellar mass deficit and merger rate for NGC 5322. In Section 4.3, we discuss the formation of NGC 5322. Section 5 summarizes our main conclusions.

1 http://leda.univ- lyon1.fr 

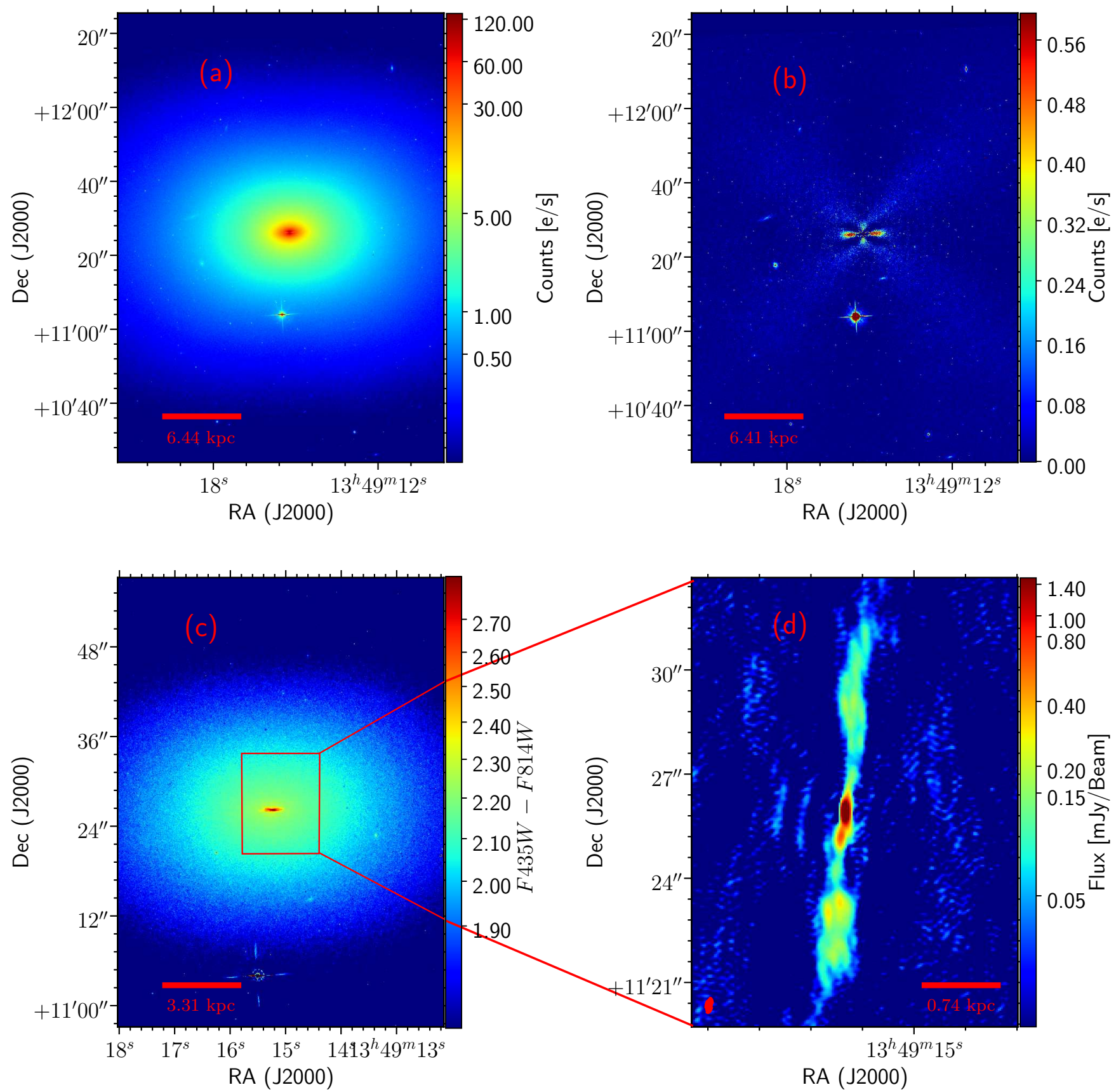

Figure 1. (a) HST ACS F814W image of NGC 5322. (b) ACS $F 814 W$ residual image of NGC 5322 created by subtracting the ELLIPSE model image from the ACS $F 814 W$ image. (c) $H S T$ ACS $F 435 W-F 814 W$ colour map of the NGC 5322 . The relatively redder $(F 435 W-F 814 W \gtrsim 2.3)$ nuclear regions are due to the nuclear dust disc with radius $R \sim 1$ !'7 $\approx 246.5 \mathrm{pc}$ and (d) e-MERLIN $1.5 \mathrm{GHz}$ radio continuum map of NGC 5233. The beam is shown in the lower left corner. In all the panels north is up, and east is to the left. The nuclear dust disc is embedded in the small-scale stellar disc with a half-light radius $R_{\mathrm{e}} \sim 356.7$ pc. Both the dust and stellar discs are edge-on and aligned along the major axis of the galaxy, i.e., in the east-west direction. The dark regions, bright cones along the major-axis and the ' $\mathrm{X}$ '-shaped structure in the residual image are due to the dust disc, stellar disc and boxy spheroid, respectively. 


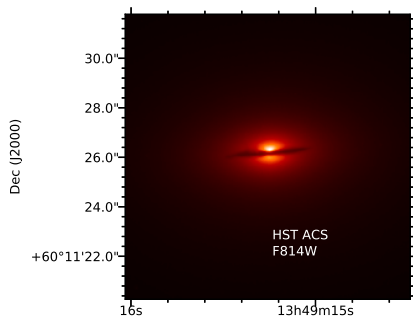

RA (12000)

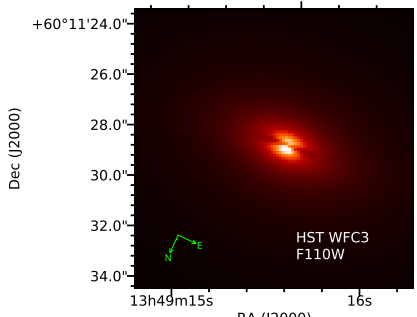

RA (12000)
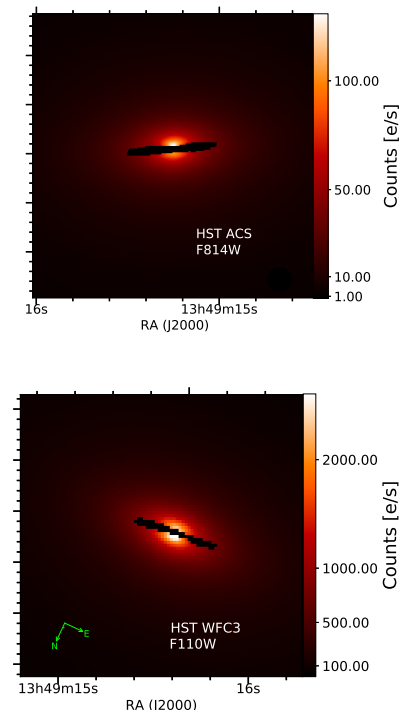

Figure 2. Top panels: HST ACS F814W image of NGC 5322 showing the central regions of the galaxy with the nuclear dust disc (left) and the corresponding masked regions (black areas) overplotted on the image (right). North is up and east is to the left. Bottom panels: as in the top panels but for the HST WFC3 F110W image. The orientation of the WFC3 images is marked with arrows.

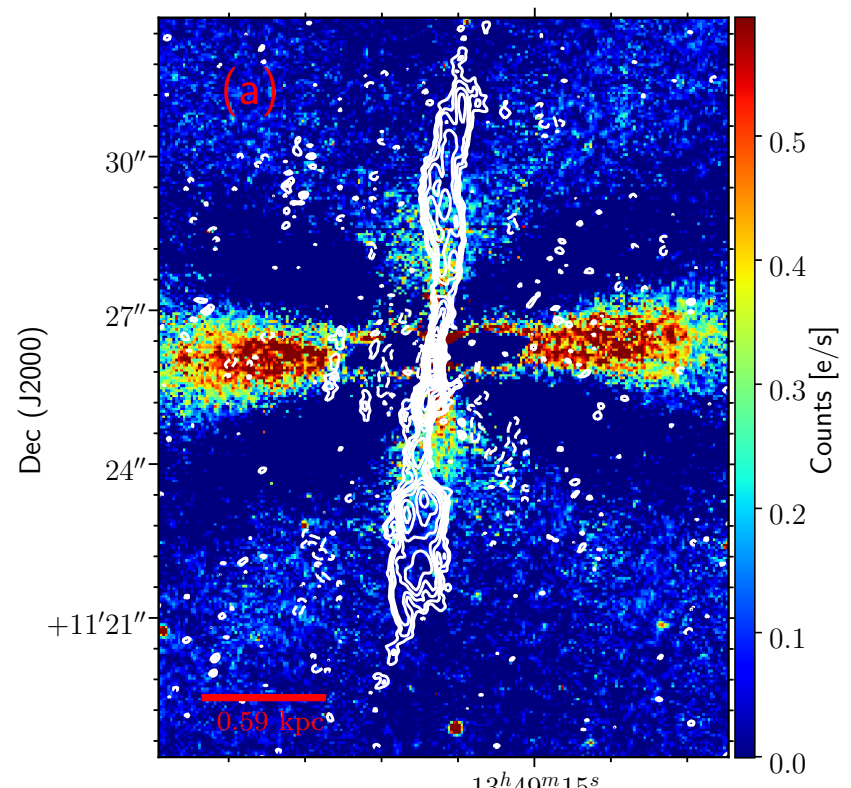

RA (J2000)

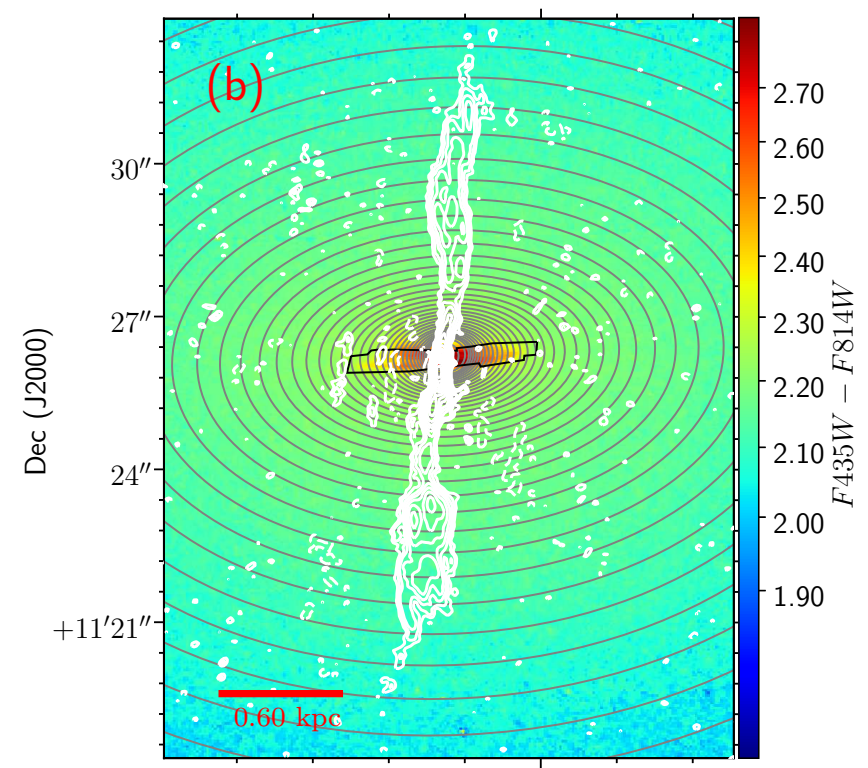

RA (J2000)

Figure 3. e-MERLIN $1.5 \mathrm{GHz}$ contours of NGC 5322 overlaid on the (a) residual image and (b) $F 435 W-F 814 W$ colour map of the galaxy, highlighting the residual structures and colour excess due to the dust disc. We overplot the elliptical isophotes derived from the ELLIPSE fit to the ACS F814W image (see Section 2.4) to illustrate the position angle of the galaxy relative to the dust disk and radio jets. For clarity we do not show a few isophotes inside $R \leq 0^{\prime \prime} .4$. While the black contour (with a semi-major axis of $\sim 11^{\prime \prime} 7$ ) encloses the masked region shown in Fig. 2, we use the ACS F814W light profile at $3^{\prime \prime} \lesssim R \lesssim 40^{\prime \prime}$ (Section 2.4). The radio jet is nearly perpendicular to the nuclear stellar and dust discs that are aligned along the major axis of the galaxy and result in the bright cones in East-West direction (a) and the colour excess (b), respectively. In both panels north is up, and east is to the left. The contour levels are (-1.00, 1.00, $1.41,2.00,2.83,4 ., 5.66,8.00,11.31,16.00,22.63,32.00,45.25,64.00,90.51,128.00,181.00,256.00,362.00$ and 512.00$) \times 3.326 \mathrm{E}-05 \mathrm{Jy}$ beam $^{-1}$. 


\section{OBSERVATIONS AND DATA REDUCTION}

\section{1 e-MERLIN radio data}

High-sensitivity, high-resolution radio continuum observations of NGC 5322 were carried out using the e-MERLIN array at L-band (1.25-1.75 GHz) on 17-May-2015 as part of the e-MERLIN LeMMINGs legacy programme (Beswick et al. 2014). We used all seven of the e-MERLIN telescopes throughout these observations, including the 76-m Lovell telescope. The target source was observed for a total on-source time of $5.7 \mathrm{hrs}$. These observations were regularly interspersed with short scans of the nearby phase calibration source, J1335+5844, with a 13.2 minute target to phase calibrator cycle. Observations of flux density calibration source, 3C286, and bandpass calibrator, OQ208, were made at the beginning and end of the observing run. A total bandwidth of $512 \mathrm{MHz}$ was correlated into 8 adjacent spectral windows with each spectral window correlated into 512 frequency channels per polarisation. Following initial data editing and the excision of RFI these data were averaged in frequency $(0.5 \mathrm{MHz})$ and time (2 sec) before being processed using the e-MERLIN pipeline (Argo 2015) to apply standard multi-frequency calibration procedures to determine and apply the complex gain solutions derived from the calibration sources. Following these pipeline procedures a number of self-calibration cycles were carried out on the target source, NGC 5322, before reweighting of the relative sensitivities of the individual e-MERLIN telescopes (as a function of time and frequency) and final imaging.

Multi-frequency synthesis imaging techniques were applied to the calibrated target source data using a variety of weighting schemes optimising for sensitivity and angular resolution. The final images (see Fig. $1 \mathrm{~d}$ ) with a restoring beam of $0^{\prime \prime} .43 \times 0$.' 17 , $\mathrm{a} \sim 41 \times 41 \mathrm{arcsec}^{2}$ field-of-view (FOV), rms noise level of $11.6 \mu \mathrm{Jy}_{\text {beam }}{ }^{-1}$ and peak target flux density of $5.26 \mathrm{mJy}^{\mathrm{beam}}{ }^{-1}$ were used for subsequent analysis.

\section{$2.2 \quad H S T$ imaging data}

HST Advanced Camera for Surveys (ACS; Ford et al. 1998) Wide Field Channel (WFC) $F 435 W$ and $F 814 W$ images of NGC 5322 (HST Proposal ID 9427, Harris et al. 2006) were retrieved from the public Hubble Legacy Archive (HLA ${ }^{2}$ ). The ACS $F 435 W$ and $F 814 W$ filters are similar to Johnson's $B$ - and $I$-bands, respectively. In addition, we retrieved the $H S T$ Wide Field Camera 3 (WFC3) IR F110W image from the Mikulski Archive for Space Telescopes (MAST ${ }^{3}$ ) to better account for the edge-on nuclear dust disc visible in the images and in our colour map (Figs. 1, 2 and 3). The ACS WFC and WFC3 IR cameras have pixel scales of $0 .{ }^{\prime \prime} 05$ pixel $^{-1}$ and $0^{\prime \prime} .13$ pixel $^{-1}$, respectively.

\subsection{SDSS imaging data}

We obtained the SDSS $i$-band mosaic image of NGC 5322, with a pixel scale of $0^{\prime \prime} 396$ and a $\sim 18 \times 18$ arcmin $^{-2}$ FOV from the Data Release $13\left(\mathrm{DR} 13^{4}\right)$ database. This image was used to determine reliably the sky background of NGC 5322.

\subsection{Surface brightness profile}

We follow the reduction procedure by Dullo et al. (2016, see references therein) and fit elliptical isophotes to the WFC3 $F 110 W$, ACS $F 814 W$ and SDSS $i$-band images of NGC 5322 using the IRAF task ELLIPSE (Jedrzejewski 1987) to extract the surface brightness, ellipticity $(\epsilon=1-\mathrm{b} / \mathrm{a}$, where $\mathrm{a}$ and $\mathrm{b}$ are the semi-major and minor-axes of the best-fit ellipse), position angle (P.A.) and isophote shape parameter $\left(B_{4}\right)$ profiles along the major-axis (see Fig. $3 \mathrm{~b}$ ). $B_{4}$ quantifies deviations of the isophotes from pure ellipses (i.e., $B_{4}=0$ ). If $B_{4}<0$ then the isophotes are boxy, $B_{4}>0$ if the isophotes are discy. We run ELLIPSE on the WFC3 $F 110 W$, ACS $F 814 W$ and SDSS $i$-band images using a mask for each image that is generated by SExtractor (Bertin \& Arnouts 1996) together with a manual mask to exclude the galaxy's nuclear dust disc, gaps between CCD detectors in the images, background galaxies, bright foreground stars, and chip defects.

Fig. 4 shows a composite light profile of NGC 5322 together with the pertaining $\epsilon$, P.A. and $B_{4}$ profiles, assembled by combining our WFC3 $F 110 W$, ACS $F 814 W$ and SDSS $i$-band data. At $R \lesssim 3^{\prime \prime}$, we used the near-IR WFC3 $F 110 W$ light profile which is significantly less contaminated by dust extinction (see Sections 3.2 and 4.2.1). In Fig. 2, we show the central regions and associated masks of NGC 5322 to highlight the masked nuclear dust regions in the WFC3 F110W and ACS $F 814 W$ images. The ACS $F 814 W$ data, with a better spatial resolution than the WFC3 and SDSS data, were used at $3^{\prime \prime} \lesssim R \lesssim 40^{\prime \prime}$. In order to capture NGC 5322's stellar halo that extends beyond the FOVs of the ACS and WFC3 images, at large radii $\left(R \gtrsim 40^{\prime \prime}\right)$ we used the SDSS $i$-band data to constrain the sky-background. The WFC3 F110W and SDSS $i$-band data were shifted to match the ACS $F 814 W$ data. Although the WFC3 $F 110 W$ filter covers a wavelength range mostly redder

2 https://hla.stsci.edu/hlaview.html

3 https://archive.stsci.edu

4 http://www.sdss.org/dr13/ 


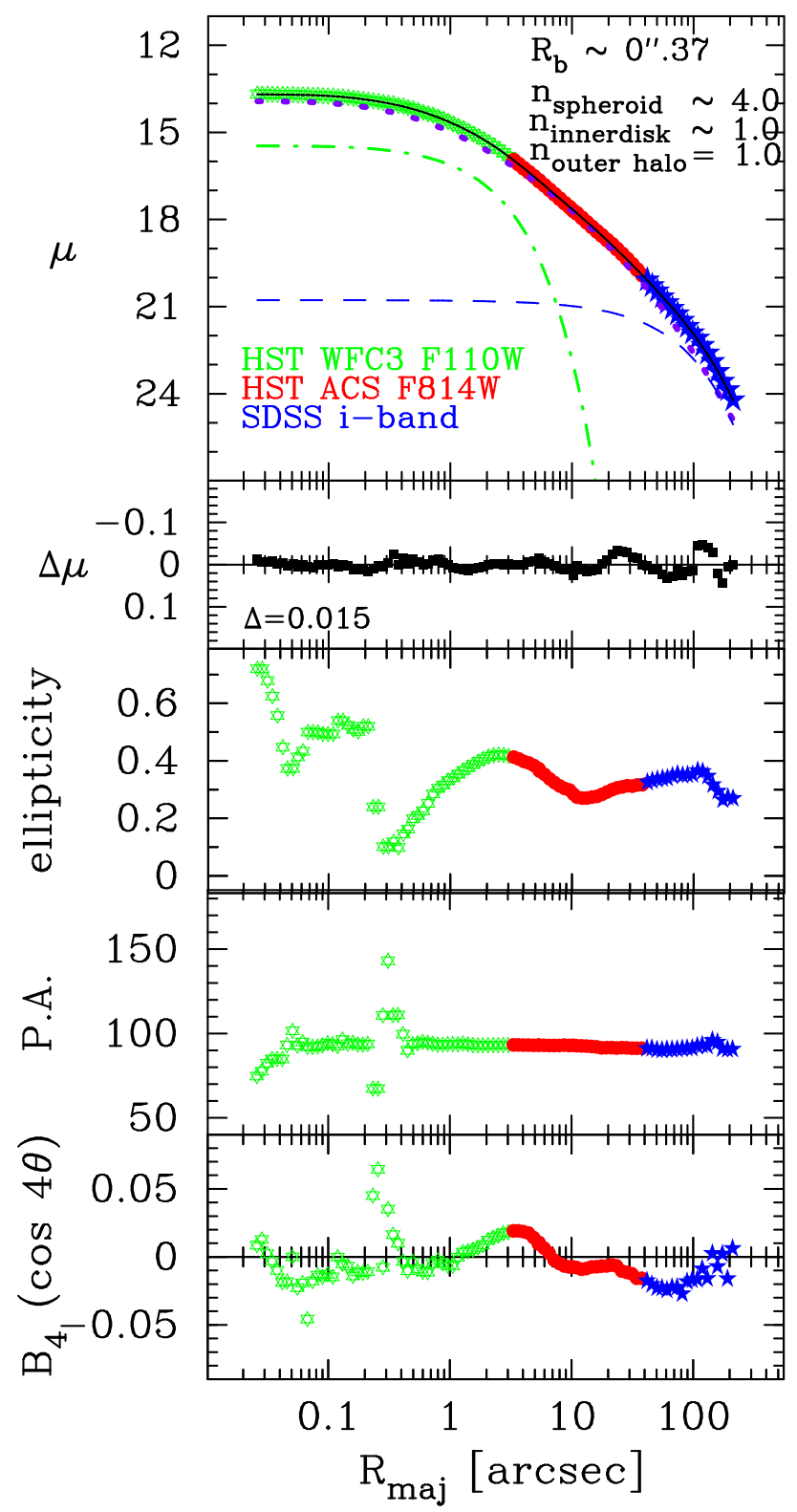

Figure 4. Three-component inner disc (dash-dotted green curve) + boxy spheroid (dotted magenta curve) + outer halo (dashed blue curve) model fit to the composite HST WFC3 IR F110W + HST ACS F814W + SDSS $i$-band surface brightness profile of NGC 5322 (see Section 2.4). The solid black curve shows the complete fit to the profile. The WFC3 $F 110 W$ and SDSS $i$-band light profiles were shifted to match the ACS $F 814 W$ data. This decomposition yields a depleted core with a break radius of $R_{\mathrm{b}} \sim 00^{\prime \prime} 37 \pm 0^{\prime \prime} 04$. Note that we extracted the innermost $\left(R \lesssim 3^{\prime \prime}\right)$ light profile of the galaxy using (i) the WFC3 IR F110W image and (iii) a careful mask to avoid the nuclear dust disc, with a thickness of $\sim 00^{\prime \prime} 2 \approx 28$ pc near the center of the galaxy, dividing the galaxy centre (Fig. 2).

than those of the ACS $F 814 W$ - and SDSS $i$-band filters, we find a good overlap between the ACS $F 814 W$, SDSS $i$ and WFC3 $F 110 W$ light profiles over the $R \lesssim 10^{\prime \prime}$ radial range, when we exclude the dust-affected region. The magnitudes in this paper are in the VEGA magnitude system.

\section{STRUCTURAL ANALYSIS}

\subsection{Residual image and dust map}

Fig 1(b) shows the residual image of NGC 5322 generated by subtracting the IRAF ELLIPSE model image from the ACS $F 814 W$ image. Four structural features are evidenced in this image: (i) a dark nuclear disc along the galaxy's major axis which is due to the edge-on inner dust disc (with a radius of $1^{\prime \prime} .7 \approx 246.5 \mathrm{pc}$ and a thickness of $0 .{ }^{\prime \prime} 6 \approx 87 \mathrm{pc}$ near the galaxy centre, respectively) that is visible in the galaxy images crossing the centre of the galaxy, (ii) two bright cones along the 
A high-resolution, multi-band analysis of NGC 5322.

Table 1. Structural parameters for NGC 5322 resulting from the fit to the optical data

\begin{tabular}{|c|c|c|c|c|c|c|c|c|c|c|c|c|c|c|c|c|}
\hline $\begin{array}{l}\text { Galaxy } \\
\text { (1) }\end{array}$ & $\begin{array}{l}\text { Type } \\
(2)\end{array}$ & $\begin{array}{l}\mu_{\mathrm{b}} \\
(3)\end{array}$ & $\begin{array}{c}R_{\mathrm{b}} \\
(\operatorname{arcsec}) \\
(4)\end{array}$ & $\begin{array}{c}R_{\mathrm{b}} \\
(\mathrm{pc}) \\
(5)\end{array}$ & $\begin{array}{c}\gamma \\
(6)\end{array}$ & $\begin{array}{l}\alpha \\
(7)\end{array}$ & $\begin{array}{l}n_{\mathrm{CS}} \\
(8)\end{array}$ & $\begin{array}{c}R_{\mathrm{e}, \mathrm{cS}} \\
(\operatorname{arcsec}) \\
(9)\end{array}$ & $\begin{array}{c}R_{\mathrm{e}, \mathrm{cS}} \\
(\mathrm{kpc}) \\
(10)\end{array}$ & $\begin{array}{l}\mu_{\mathrm{e}, \mathrm{S}} \\
(11)\end{array}$ & $\begin{array}{l}n_{S} \\
(12)\end{array}$ & $\begin{array}{c}R_{\mathrm{e}, \mathrm{S}} \\
(\operatorname{arcsec}) \\
(13)\end{array}$ & $\begin{array}{l}R_{\mathrm{e}, \mathrm{S}} \\
(\mathrm{pc}) \\
(14)\end{array}$ & $\begin{array}{c}\mu_{0, \text { halo }} \\
(15)\end{array}$ & $\begin{array}{c}h \\
(\operatorname{arcsec}) \\
(16)\end{array}$ & $\begin{array}{c}h \\
(\mathrm{kpc}) \\
(17)\end{array}$ \\
\hline NGC 5322 & E3-4 & 14.36 & $\begin{array}{l}0.37 \\
10 \%\end{array}$ & 53.7 & $\begin{array}{l}0.17 \\
10 \%\end{array}$ & 2 & $\begin{array}{c}4.0 \\
20 \%\end{array}$ & $\begin{array}{l}30.4 \\
25 \%\end{array}$ & 4.4 & 17.21 & $\begin{array}{c}1.0 \\
20 \%\end{array}$ & $\begin{array}{l}2.46 \\
20 \%\end{array}$ & 356.7 & 20.79 & $\begin{array}{l}53.6 \\
10 \%\end{array}$ & 7.8 \\
\hline
\end{tabular}

Notes.- First row: structural parameters from the three-component Sérsic inner disc + core-Sérsic spheroid + exponential halo fit to the $F 814 W$-band ( $\sim$-band) surface brightness profiles of NGC 5322 (Fig. 4). Col. 1: galaxy name. Col. 2: morphological type. Cols. 3-10: best-fit parameters from the core-Sérsic model. Cols. 11 - 14: Sérsic model parameters for the exponential $(n=1)$ inner stellar disc with scale length $\left(h=R_{\mathrm{e}, \mathrm{S}} / 1.678\right) \sim 1^{\prime \prime}$. 47 and central surface brightness $\left(\mu_{0, \mathrm{disc}}\right) \sim \mu_{\mathrm{e}, \mathrm{S}}-1.822 \sim 15.39 \mathrm{mag} \operatorname{arcsec}^{-2}$. Cols. $15-17$ best-fit parameters for the exponential halo light. $\mu_{\mathrm{b}}, \mu_{\mathrm{e}}$ and $\mu_{0, \text { halo }}$ are in mag $\operatorname{arcsec}^{-2}$. The second row shows the uncertainties on the fit parameters. The uncertainty associated with $\mu_{\mathrm{b}}, \mu_{\mathrm{e}, \mathrm{S}}$ and $\mu_{0, \mathrm{~h}}$ is $\sim 0.02 \mathrm{mag} \operatorname{arcsec}^{-2}$.

major axis, (ii) two bright cones along the minor-axis and (iv) a large-scale ' $\mathrm{X}$ '-shaped structure due to the spheroid's boxy isophotes (see also Fig. 3a and Section 3.4). The dust disc, with $F 435 W-F 814 W \sim 2.72_{-0.39}^{+0.13}$ compared to the relatively bluer immediate surroundings $(F 435 W-F 814 W \sim 2.30)$, is better visible in the colour maps (Figs. 1c and $3 \mathrm{~b}$ ), rather than in the residual image, as an inner disc with red colour excess of $\sim 0.42$ mag (see also Carollo et al. 1997; Tomita et al. 2000). To create this colour map, we registered the $F 435 W$ and $F 814 W$ images using the IRAF tasks GEOMAP and GREGISTER. Excluding the dust disc, the galaxy becomes progressively bluer toward larger radii, with an $F 435 W-F 814 W$ colour gradient of $\sim 0.22 \mathrm{mag}$ per $10^{\prime \prime}$. We note that the dust disc has a thickness of $\sim 00^{\prime \prime} 2 \approx 28$ pc near the galaxy center in the WFC3 F110W image (Fig. 2).

\subsection{Structural decomposition}

NGC 5322 is well known for its inner (discy) stellar component, which counter-rotates with respect to the galaxy's boxy spheroid (e.g., Bender 1988, 1996; Bender \& Surma 1992; Rix \& White 1992; Goudfrooij et al. 1994; Scorza \& Bender 1995; Carollo et al. 1997; Silva \& Bothun 1998), and for its central radio source (Feretti et al. 1984; Hummel et al. 1984). Analysis of high-resolution HST ACS/WFC images shows that the galaxy contains a small-scale discy stellar component, a boxy spheroid (that has a depleted core as revealed by the flattened inner stellar distribution which deviates downward relative to the inward extrapolation of the spheroid's outer Sérsic profile) and an outer stellar halo (Figs. 1, 3 and 4). As mentioned in the Introduction, early-type galaxies brighter than $M_{B} \sim-20.5 \mathrm{mag}$, hereafter core-Sérsic galaxies, have depleted cores and central stellar mass deficits which are thought to be created through the ejection of stars by inspiralling binary SMBHs (not due to central dust obscuration), while those with $M_{B} \gtrsim-20.5$ mag tend to be coreless (e.g., Faber et al. 1997; Kormendy 1999; Rest et al. 2001; Ravindranath et al. 2001; Trujillo et al. 2004; Lauer et al. 2005, 2007; Ferrarese et al. 2006; Dullo \& Graham 2012, 2013, 2014; Rusli et al. 2013).

The light distributions of core-Sérsic galaxies are well described by the core-Sérsic model ${ }^{5}$ which is a blend of an inner power-law and an outer Sérsic model (Graham et al. 2003). Detailed multicomponent decompositions using high-resolution light profiles, extending to at least $1 R_{\mathrm{e}}$ (Graham et al. 2003), are crucial to understand properly the different formation mechanisms that build up the distinct structural components in galaxies. Therefore, we fit a three-component Sérsic smallscale disc + core-Sérsic spheroid + exponential halo model to the major-axis surface brightness profile of NGC 5322 which extends out $200^{\prime \prime}$. Fig. 4 shows this light profile decomposition with a small $\mathrm{rms}$ residual scatter of $\sim 0.015 \mathrm{mag}^{\mathrm{arcsec}}{ }^{-2}$. Each model component was convolved with a Gaussian PSF with FWHM $\sim 0$ ". 25 (see Dullo, Graham, \& Knapen 2017, their Section 3.1). The FWHM of the PSF was determined using several stars in the WFC3 F110W image of the galaxy. Table 1 provides the best-fitting model parameters.

We found that a Sérsic model fit to the halo light yields $n \sim 1.0 \pm 0.2$. We therefore force an exponential $(n=1)$ model for the outer stellar halo. Similarly, the embedded, small-scale discy component was modelled using a Sérsic model with a half-light effective radius $R_{\mathrm{e}} \sim 2^{\prime \prime} .46 \pm 0$.' $49 \approx 356.7 \pm 71 \mathrm{pc}$ and $n \sim 1.0$, indicating an inner exponential disc with a scale length $h \sim 1^{\prime \prime} .47 \pm 0^{\prime \prime} .15 \approx 212.6 \pm 21.3 \mathrm{pc}$ and an $I$-band central surface brightness $\mu_{0, \text { disc }} \sim 15.39 \pm 0.02 \mathrm{mag} \operatorname{arcsec}^{-2}$. The boxy spheroid which dominates over $R \lesssim 100^{\prime \prime}$ has a core-Sérsic stellar light distribution with $n \sim 4.0 \pm 0.8$ (i.e., essentially the de Vaucouleurs (1948) $R^{1 / 4}$ law outside the depleted core), $R_{\mathrm{b}} \sim 0^{\prime \prime} 37 \pm 00^{\prime \prime} 04, \mu_{\mathrm{b}, I} \sim 14.36 \pm 0.02 \mathrm{mag}^{\mathrm{arcsec}}{ }^{-2}$ and $^{\prime}$ $R_{\mathrm{e}} \sim 30^{\prime \prime} 4 \pm 7^{\prime \prime}$. 6 . At $R \gtrsim 100^{\prime \prime}$, the spheroid and outer stellar halo contribute equally.

5 Dullo \& Graham $(2012,2014)$ discussed the core-Sérsic model in detail and provided a comparison between the model and the Nuker model (Lauer et al. 1995), see also (Graham et al. 2003; Trujillo et al. 2004; Ferrarese et al. 2006). 


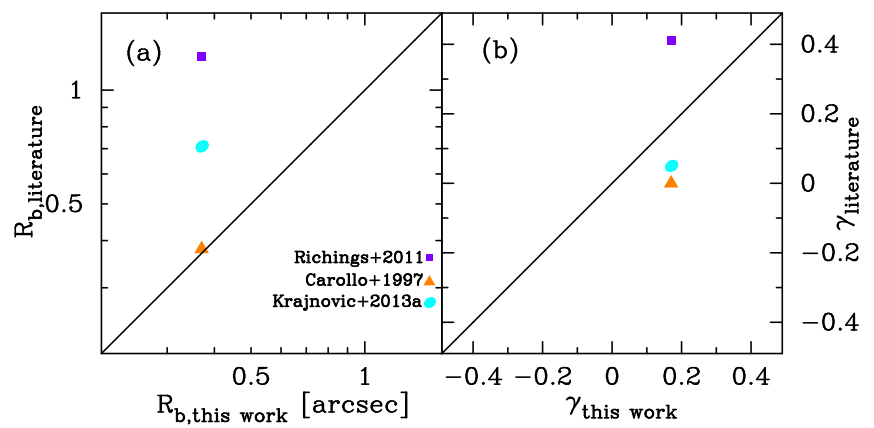

Figure 5. Comparison of our break radius $\left(R_{\mathrm{b}}\right)$ and inner logarithmic slope of the spheroid's inner light profile $(-\gamma)$ with those values from Carollo et al. (1997), Richings et al. (2011) and Krajnović et al. (2013a). The solid lines show a one-to-one relation.

\subsection{Literature comparison}

For comparison, Rix \& White (1992) decomposed the radially limited $\sim 10^{\prime \prime}$ absorption line profile of NGC 5322, which does not probe the outer parts of this galaxy's spheroid and stellar halo, into an inner stellar disc with $h \sim 2$.' 1 and a spheroid with $R_{\mathrm{e}} \sim 25^{\prime \prime}$ using a double Gaussian model. Direct comparison between our decomposition and that by Rix \& White (1992) is challenging given the different approaches by these two works, i.e., absorption line profile vs. light profile decomposition. Scorza \& Bender (1995, their Figure A.9) fit an exponential disc $+R^{1 / 4}$ bulge model to their $\sim 10^{\prime \prime}$ data with a seeing FWHM of $1^{\prime \prime} .6$ and a plate scale of 0 .' $46 /$ pixel, finding $R_{\mathrm{e}} \sim 3.4 \mathrm{kpc}$ for the bulge, and $h \sim 180 \mathrm{pc}$, a $V$-band $\mu_{0, \text { disc }} \sim 19.45$ mag $\operatorname{arcsec}^{-2}$.

Fig. 5 compares our break radius $\left(R_{\mathrm{b}}\right)$ and inner logarithmic slope of the spheroid's inner light profile $(-\gamma)$ with those from Carollo et al. (1997), Richings et al. (2011) and Krajnović et al. (2013a). Carollo et al. (1997) and Krajnović et al. (2013a) fit the Nuker model (Lauer et al. 1995, 2005) to 10" HST WFPC2/PC1 light profile of NGC 5322, while Richings et al. (2011) modelled the galaxy's HST ACS/WFC light profile using the core-Sérsic model. Also, Krajnović et al. (2013b, their Fig. 1) modelled their ground based light profile of the galaxy over the radius range $2 . ' 5 \lesssim R \lesssim 172^{\prime \prime}$ using a single Sérsic model, resulting in a residual profile which clearly reveals structures that have not been properly modelled. Because these past works (Carollo et al. 1997; Richings et al. 2011; Krajnović et al. 2013b,a) treated the galaxy as a single-component system, the fraction of the light attributed to the spheroid in their modellings come from the inner stellar disc and outer halo. This led, in part, the measurements of the break radius/half-light radius and Sérsic index by Richings et al. (2011, $R_{\mathrm{b}} \sim 11^{\prime \prime} 23$, $n \sim 8.1$ and $R_{\mathrm{e}} \sim 93^{\prime \prime}$, 1 , their Table 2) and Krajnović et al. (2013b, $n \sim 5.8, R_{\mathrm{e}} \sim 67^{\prime \prime}$ '9, their Fig. 1) to be biased high (Fig. 5). Measuring the core size as the radius where the negative logarithmic slope of the fitted Nuker model profile equals $1 / 2\left(R_{\gamma=1 / 2}\right)$, Krajnović et al. (2013a, their Table C1) found $R_{\gamma=1 / 2} \sim 0^{\prime \prime}$.71, while Carollo et al. (1997, their Table 4) reported $R_{\gamma=1 / 2} \sim 0^{\prime \prime} 38$ which agrees with our core-Sérsic break radius for the galaxy (Fig. 5a).

Part of the discrepancies between our work and Carollo et al. (1997), Krajnović et al. (2013a) and Richings et al. (2011) arises from the treatment of the inner dust disc. Indeed, the inner negative logarithmic slopes presented in Carollo et al. (1997, $\gamma=0.0)$, and Krajnović et al. (2013a, $\gamma=0.05)$ are shallower than that of ours $(\gamma \sim 0.17 \pm 0.02)$, while Richings et al. (2011) reported a very steep inner negative logarithmic slope for the galaxy $(\gamma=0.41$, Fig. 5b). Furthermore, replacing the inner $R \lesssim 3^{\prime \prime}$ profile in Fig. 4, extracted from the redder WFC3 $F 110 W$ image, with the ACS $F 814 W$ data and performing a 3-component decomposition of the ACS F814W $\left(R \lesssim 40^{\prime \prime}\right)+\operatorname{SDSS} i$-band $\left(R>40^{\prime \prime}\right)$ profile yields a core with $R_{\mathrm{b}} \sim$ 0 .' $49 \pm 0$. .'05 $\approx 1.0 \pm 7.3 \mathrm{pc}$, compared to that adopted here $\left(R_{\mathrm{b}} \sim 00^{\prime \prime} 37 \pm 00^{\prime \prime} 04 \approx 53.7 \pm 5.4\right.$ pc, see Fig. 4 and Table 1$)$. In Section 4.2 .1 , we measure low $F 110 W$-band extinction $\left(A_{F 110 W} \sim 0.031-0.047 \mathrm{mag}\right)$ in the regions immediately surrounding the masked regions near the galaxy centre (Figs. 2 and 3). Also, we show that our break radius for this galaxy is in good agreement with those predicted based on the Dullo \& Graham $(2014) R_{\mathrm{b}}-\sigma$ and $R_{\mathrm{b}}-M_{V}$ relations, i.e., $R_{\mathrm{b}, \sigma-\text { based }} \approx 44.0 \pm 6.1$ pc and $R_{\mathrm{b}, M_{V} \text {-based }} \approx 48.6 \pm 7.2 \mathrm{pc}$, respectively (Section 4.2 ). This $R_{\mathrm{b}}$ agreement, together with the low IR extinction, gives us some confidence that our measurement of NGC 5322's break radius was not affected by the nuclear dust disc.

\subsection{Ellipticity, Position Angle, Isophote Shape and Kinematics}

Inside $R \sim 0$ "' 3 , NGC 5322 shows radial variations in ellipticity, P.A. and $B_{4}$ due to the nuclear dust disc and the PSF (Figs. 1 and 4). Going outward from $R \sim 0^{\prime \prime} 3$, the ellipticity continuously rises from $\epsilon \sim 0.10$ to a maximum of $\epsilon \sim 0.45$ at $\sim 2^{\prime \prime} .5$ and it then declines reaching a local minimum of $\sim 0.25$ at $\sim 10^{\prime \prime}$, before starting to gently rise again. The ellipticity profile together with the positive $B_{4}$ values over $1^{\prime \prime} .5-8^{\prime \prime}$ reveal the small-scale disc, consistent with the 3-component profile decomposition (Fig. 4). The spheroid of NGC 5322 contains boxy isophotes, with ellipticity $\epsilon \sim 0.35 \pm 0.07$, that resulted in the 'X'-shaped structure in the residual image (Figs. 1 and 3). Bender (1988, see also Rix \& White 1992; Scorza \& Bender 1995 and Krajnović et al. 2013b) found that the inner region $\left(R \lesssim 2^{\prime \prime} .6\right)$, where this galaxy's inner disc contributes significantly, 
Table 2. e-MERLIN observation

\begin{tabular}{|c|c|c|c|c|c|c|c|c|c|c|}
\hline $\begin{array}{l}\text { Frequency } \\
(\mathrm{GHz}) \\
(1)\end{array}$ & $\begin{array}{l}\theta_{\mathrm{maj}} \times \theta_{\mathrm{min}} \\
(2)\end{array}$ & $\begin{array}{l}\text { Beam P.A. } \\
\left({ }^{\circ}\right) \\
(3)\end{array}$ & $\begin{array}{c}\theta_{\mathrm{maj}} \times \theta_{\mathrm{min}} \\
(4)\end{array}$ & $\begin{array}{c}\text { Beam P.A. } \\
\left({ }^{\circ}\right) \\
(5)\end{array}$ & $\begin{array}{c}S_{\text {peak }} \\
\left(\text { mJy beam }^{-1}\right) \\
(6)\end{array}$ & $\begin{array}{c}S_{\text {int,core }} \\
(\mathrm{mJy}) \\
(7)\end{array}$ & $\begin{array}{c}T_{\mathrm{B}, \text { core }} \\
(\mathrm{K}) \\
(8)\end{array}$ & $\begin{array}{c}P_{\text {core }} \\
\left(\mathrm{W} \mathrm{Hz}^{-1}\right) \\
(9)\end{array}$ & $\begin{array}{c}S_{\text {int,jets }} \\
(\mathrm{mJy}) \\
(10)\end{array}$ & $\begin{array}{c}P_{\text {jets }} \\
\left(\mathrm{W} \mathrm{Hz}^{-1}\right) \\
(11)\end{array}$ \\
\hline$\overline{1.5}$ & 0. & 0.79 & $0 .{ }^{\prime \prime} 20 \times 0 . .04$ & $169.6 \pm 0.094$ & $5.38 \pm 0.01$ & $5.96 \pm 0.02$ & $4.5 \times 10^{7}$ & $6.55 \times 10^{20}$ & $6.41 \pm 0.02$ & $7.04 \times 10^{20}$ \\
\hline
\end{tabular}

Notes.-Col. 1: centrally weighted (i.e., nominal) frequency. Col. 2: synthesised beam major- and minor-axis dimensions. Col. 3: synthesised beam position angle. Cols. 4 and 5: deconvolved beam dimensions and position angle obtained from a single Gaussian component fit to the central radio emission using the AIPS task JMFIT. Col. 6: JMFIT peak flux density of the core emission. Col. 7: JMFIT integrated flux density of the core emission. Cols. 8 and 9: brightness temperature and power of the radio core. Col. 10: integrated flux density of the jets. Col. 11: radio power of the jets. The quoted errors on the P.A., $S_{\text {peak }}$ and $S_{\text {int,core }}$ (Cols. 5,6 , and 7 ) are those reported by the JMFIT. The error on $S_{\text {int,jets }}$ is that given by TVSTAT.

counter-rotates with maximum rotational velocity $V_{\text {rot,max }} \sim 80 \mathrm{~km} \mathrm{~s}^{-1}$ relative to the intermediate parts the spheroid at $10^{\prime \prime} \lesssim R \lesssim 35^{\prime \prime}\left(V_{\text {rot,max }} \sim-30 \mathrm{~km} \mathrm{~s}^{-1}\right)$. Rix \& White (1992, their Fig. 9) reported $V_{\text {rot,max }} / \sigma \sim 1.4$ and $\sim 0.15$ for the inner disc and spheroid, respectively.

The small-scale stellar disc, spheroid and outer stellar halo of NGC 5322 are well aligned as revealed by the position angle which remains stable at $\sim 94^{\circ}$ outside the region most affected by the PSF (see Fig. 4).

\subsection{Radio structure}

NGC 5322 is a radio source which hosts a LINER nucleus (Baldi \& Capetti 2009). Fig. 1(d) shows the 1.5 GHz e-MERLIN radio continuum map of NGC 5322 with a restoring beam of $\theta_{\text {maj }} \times \theta_{\min } \sim 0^{\prime \prime} 43 \times 00^{\prime \prime} 17$ at a P.A. $\sim 0.79^{\circ}$ (Table 2). Lower resolution images of this source in the Faint Images of the Radio Sky at Twenty Centimeters (FIRST) survey (Becker, White, \& Helfand $1995)$ at $1.4 \mathrm{GHz}$ show that it has an extended radio jet structure along the same position angle of the inner jet structure observed in Fig 1. Whilst the VLA maps of the galaxy by Hummel et al. (1984) also revealed that the galaxy has an FRI radio morphology with twin radio jets extending over $\sim 20^{\prime \prime}$ at $1.4 \mathrm{GHz}$. These results are consistent but substantially shallower and at lower angular resolution, as compared to these new e-MERLIN observations. While the large bandwidth of these observations provides near complete aperture coverage to emission on scales between 0.1 to several arcsec, it should be noted that the shortest project baseline of e-MERLIN $(\sim 11 \mathrm{~km})$ in these observations means that these data are insensitive to very extended, diffuse emission. In the case of NGC 5322 this spatial filtering acts to separate the central jet emission from diffuse radio emission. Fig. $1(\mathrm{~d})$ shows that these radio jets extend $\sim 10^{\prime \prime} .9 \approx 1.6 \mathrm{kpc}$ at a position angle of $172^{\circ}$ as observed with e-MERLIN. On large scales, the radio jets show slight deviations with respect to the orientation of the inner jet.

Fig. 3 shows the radio contours overlaid on the HST ACS residual image and the ACS $F 435 W-F 814 W$ colour map. In Fig. 3(b), we overplot the elliptical isophotes obtained from the ellipse fit to the ACS F814W image to illustrate the position angle of the galaxy relative to the nuclear dust disk and radio jets. Because of NGC 5322's nuclear dust disc crossing the galaxy centre, we did not attempt to align these images by shifting the radio core to the central maximum in the ACS images. We determine that the offset between the optical and radio images can be up to $\sim 0{ }^{\prime \prime} 3$. The inner jet is nearly perpendicular to the nuclear dust and stellar discs that are aligned along the major axis of the galaxy (i.e., P.A. $\sim 94^{\circ}$ ). The radio jets do not have strong enough optical/near-IR emissions to be seen from the optical and near-IR images, or as excess optical/IR emission above the (inner disc + spheroid + halo) model fit to the galaxy light profile (Fig 4).

\subsubsection{Flux density, brightness temperature and radio power}

We fit a single elliptical Gaussian model to the central radio source using the AIPS ${ }^{6}$ task JMFIT, deriving the position, peak and integrated intensity flux density of the core emission. Also, the JMFIT fit yields deconvolved beam dimensions and position angle after deconvolving the clean beam from the fitted component size (see Table 2).

The measurement of the brightness temperature of a radio source is a diagnostic tool that discriminates between the thermal free-free emission from $\mathrm{H}$ II regions and non-thermal synchrotron radio continuum emission. The former has a nebular brightness temperature of $T_{\mathrm{B}} \sim 10^{4} \mathrm{~K}$, while the latter has a higher brightness temperature $T_{\mathrm{B}} \gtrsim 10^{6}-10^{11} \mathrm{~K}(\mathrm{e} . \mathrm{g}$, Condon 1992; Ho \& Ulvestad 2001).

\footnotetext{
6 http://www.aips.nrao.edu/
} 
Using the Rayleigh-Jeans Law, the brightness temperature $\left(T_{\mathrm{B}}\right)$ can be written as

$T_{\mathrm{B}}=\left(\frac{S_{\nu}}{\Omega_{\mathrm{beam}} \nu^{2}}\right)\left(\frac{c^{2}}{2 k}\right)$

where $\nu$ is the central frequency, $k$ is the Boltzmann constant, $\mathrm{c}$ is the speed of light, $\Omega_{\mathrm{beam}}$ is the deconvolved beam solid angle and $S_{\nu}$ is the integrated radio flux density. For the radio core of NGC 5322 we derived $T_{\mathrm{B}} \sim 4.5 \times 10^{7} \mathrm{~K}$ at $\nu \sim$ $1524 \mathrm{MHz}$, suggesting a non-thermal radio continuum emission. This confirms the conclusion from VLA 15 GHz and VLBA observations presented by Nagar, Falcke, \& Wilson (2005) who find a brightness temperature of $>10^{8.2} \mathrm{~K}$.

We run the AIPS task TVSTAT to determine the integrated radio flux density for the jets $S_{\text {int,jets }} \sim 6.41 \pm 0.02$ mJy, which yields a $1.5 \mathrm{GHz}$ radio power of $\sim 7.04 \times 10^{20} \mathrm{~W} \mathrm{~Hz}^{-1}$ at NGC 5322's distance of $30.3 \mathrm{Mpc}$ (Table 2). Similarly, the core radio continuum emission of $S_{\text {int,core }} \sim 5.96 \pm 0.02 \mathrm{mJy}$ for NGC 5322 implies a core radio power of $\sim 6.55 \times 10^{20} \mathrm{~W} \mathrm{~Hz}^{-1}$. The NRAO VLA Sky Survey (NVSS, Condon et al. 1998), with a very poor resolution of $45^{\prime \prime}$, has detected a source, near NGC 5322's centre, with a $1.4 \mathrm{GHz}$ integrated flux of $78 \pm 2.8 \mathrm{mJy}$. The FIRST survey finds three sources with integrated radio flux densities

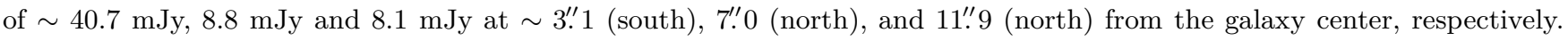
However, given the FIRST images' resolution of $5^{\prime \prime}$, this survey's detection of the three sources and the pertaining flux values for NGC 5322 are not reliable. Nonetheless, our total recovered flux density ( $S_{\text {int,jets }}+S_{\text {int,core }} \sim 12.4$ mJy) is significantly lower ( $\sim$ factor of 5 ) than that recovered for more compact/filled interferometer observations (e.g., FIRST, $S_{\text {int,total }} \sim 57.6$ mJy) due to primarily the limited number of short baselines in the e-MERLIN array which makes it insensitive to structures greater than $5^{\prime \prime}$. As such our estimate of the jet power for the galaxy is a lower limit.

\section{DISCUSSION}

\subsection{Central mass deficit}

The central stellar mass deficits of core-Sérsic galaxies are thought to be created by inspiralling binary SMBHs that form from 'dry' galaxy mergers (see Section 3.2). Theory predicts that the impact of multiple dry major mergers on core creation is cumulative. N-body simulations by Merritt (2006) suggested that the stellar mass deficit $M_{\text {def }}$ that is generated by binary SMBHs after $\mathcal{N}$ successive mergers equals $0.5 \mathcal{N} M_{\mathrm{BH}}$, where $M_{\mathrm{BH}}$ is the total sum of the masses of the binary SMBHs. A careful measurement of the central stellar mass deficit in the spheroid of NGC 5322 can be used to constrain the galaxy's merger history. We follow the procedures by Dullo \& Graham (2014, their Section 5.2) and compute the stellar luminosity deficit $\left(L_{\text {def }}\right)$ as the difference in luminosity between the inwardly-extrapolated outer Sérsic profile of the complete core-Sérsic model (fit to the spheroid's profile) and the core-Sérsic model (see Section 3.2 and Fig. 4). This yields stellar luminosity deficit in the $I$-band ${ }^{7}, L_{\text {def }} / L_{\odot, I} \sim 3.9 \times 10^{8}$. To convert this luminosity deficit into $M_{\text {def }}$, we determined the stellar mass-to-luminosity ratio $(M / L)$ excluding the inner dust disc and using the central dust-free colour of the galaxy $(F 435 W-F 814 W \sim 2.15$, Fig 1), which corresponds to $V-I \sim 1.24$ (Fukugita et al. 1995). For $V-I \sim 1.24$, the colour-age-metallicity- $(M / L)$ diagram by Graham \& Spitler (2009, their Fig. A1) yields an $M / L \sim 4.2$ in the $V$-band, which corresponds to an $I$-band $M / L \sim 2.8$ (Worthey 1994, his Table 5A), implying $M_{\text {def }} \sim(1.1 \pm 0.3) \times 10^{9} M_{\odot}$.

\subsection{Merger rate}

Dynamical SMBH measurements are not available for NGC 5322. We therefore compute the $M_{\text {def }} / M_{\mathrm{BH}}$ ratios using SMBH masses predicted from the $M_{\mathrm{BH}}-\sigma$ and $M_{\mathrm{BH}}-L$ relations. For the velocity dispersion of NGC $5322\left(\sigma \sim 229 \mathrm{~km} \mathrm{~s}{ }^{-1}\right)$, the Graham \& Scott $(2013) M_{\mathrm{BH}}-\sigma$ relation predicts $\log \left(M_{\mathrm{BH}} / M_{\odot}\right) \sim 8.51 \pm 0.40$. To estimate the total $I$-band absolute magnitude of the the spheroid $\left(M_{\mathrm{sph}, I}\right)$, we integrate the core-Sérsic profile (Fig. 4) by taking into account the spheroid's average ellipticity $(\epsilon \sim 0.35)$. This yields $M_{\mathrm{sph}, I} \sim-23.04 \mathrm{mag}$, corrected for Galactic extinction $(\sim-0.02 \mathrm{mag})$ and surface brightness dimming $(\sim-0.03 \mathrm{mag})$, which corresponds to a $V$-band magnitude $M_{\mathrm{sph}, V} \sim-21.77$ mag applying the colour transformation $V-I \sim 1.27$ (Fukugita et al. 1995). Converting the Graham \& Scott (2013) $B$-band core-Sérsic $M_{\mathrm{BH}}-L$ relation into a $V$-band relation using $B-V=0.95$ (Fukugita et al. 1995), we predict $\log \left(M_{\mathrm{BH}} / M_{\odot}\right) \sim 8.93 \pm 0.34$ for the spheroid of NGC 5322 .

We thus find that $M_{\mathrm{def}} / M_{\mathrm{BH}} \sim 3.4$ and 1.3 when using SMBH masses predicted based on the spheroid's $\sigma$ and $L$, respectively. While these numbers suggest that the spheroid has experienced $\mathcal{N} \sim 2-7$ major mergers (Merritt 2006), this conclusion is hinged on the assumption that SMBH binary scouring is the sole mechanism that created the core. For comparison, past works have found mass deficits that are typically $\sim 0.5-4 M_{\mathrm{BH}}$ (Graham 2004; Trujillo et al. 2004; Ferrarese et al. 2006; Rusli et al. 2013; Dullo \& Graham 2013, 2014). If the central mass deficit of NGC 5322 has been

7 We use an $I$-band absolute magnitude for the Sun of $M_{\odot, I} \sim 4.14 \mathrm{mag}$ (http://www.ucolick.org/ cnaw/sun.html). 
enlarged by additional mechanisms (such as the scouring action of gravitationally recoiled SMBH that oscillates about the centre of a merger remnant, e.g., Boylan-Kolchin et al. 2004; Gualandris \& Merritt 2008, and the action of multiple SMBHs that form in galaxies at high redshifts, e.g., Kulkarni \& Loeb 2012, see the discussion by Dullo \& Graham 2014, their Sections 5.3 and 5.4), then $\mathcal{N} \lesssim 2-7$.

\subsubsection{Dust absorption}

Nuclear dust absorption can reduce the surface brightnesses of NGC 5322 at the inner regions, creating an artificial depleted core or enlarging the break radius/central mass deficit and resulting in $\mathcal{N} \lesssim 2-7$ for the galaxy. However, not only did we carefully mask the inner dust disc to extract the WFC3 IR $F 110 W$ profile at $R \lesssim 3^{\prime \prime}$ (see Sections 2.4, 3.1 and Fig. 2) but also the values of $\sigma \sim 229 \mathrm{~km} \mathrm{~s}^{-1}$ and $M_{V} \sim-21.77 \mathrm{mag}$ for NGC 5322's spheroid imply that the Dullo \& Graham (2014, their Table 3) $R_{\mathrm{b}}-\sigma$ and $R_{\mathrm{b}}-M_{V}$ relations predict break radii of $R_{\mathrm{b}, \sigma-\text { based }} \approx 44.0 \mathrm{pc}$ and $R_{\mathrm{b}, M_{V}-\text { based }} \approx 48.6$ pc, in good agreement with the break radius from our modelling (Fig. 4, Table 1).

Below, as a further check on the effect of the dust absorption, we follow the dust correction steps described in Nowak et al. (2008, their Appendix A, see also Bender et al. 2015, their Section 3.3) and measure the $F 110 W\left(\sim J\right.$-band) extinction $\left(A_{J}\right)$ in the nuclear regions of the galaxy using our $F 435 W-F 814 W(B-I)$ colour map (Figs. 1 and 3 ).

Assuming that dust acts as a foreground screen obscuring the stars, the extinction in the $I$-band can be written as

$A_{I}=I_{\text {obs }}-I_{\text {corr }}=\alpha_{\text {red }} E(B-I)$,

where $I_{o b s}$ and $I_{\text {corr }}$ are the observed and extinction-corrected $I$-band surface magnitudes. The reddening $E(B-I)=$ $(B-I)_{\text {obs }}-(B-I)_{\text {corr }}$ and $\alpha_{\text {red }}=\left(\left(A_{B} / A_{I}\right)-1\right)^{-1}$. Therefore,

$f_{I, \text { corr }}=f_{I, \text { obs }}\left(\frac{f_{I, o b s}}{f_{B, o b s}}\right)^{\alpha_{\mathrm{red}}}\left(\frac{f_{B, \mathrm{corr}}}{f_{I, \text { corr }}}\right)^{\alpha_{\mathrm{red}}}$,

where $f_{I}$ and $f_{B}$ are the $I$ - and $B$-band surface fluxes.

Assuming a very shallow stellar population gradient in the central region of NGC 5322 (Spolaor et al. 2010), we found the dust free surface flux ratio in the inner regions to be $f_{B, \text { corr }} / f_{I, \text { corr }} \sim 0.125$. Following Schlafly \& Finkbeiner $(2011$, their Table 6$), A_{\mathrm{ACS}, \mathrm{F} 45 \mathrm{~W}}=3.610 E(B-V), A_{\mathrm{ACS}, \mathrm{F} 814 \mathrm{~W}}=1.526 E(B-V)$ and $A_{\mathrm{WFC} 3, \mathrm{~F} 110 \mathrm{~W}}=0.881 E(B-V)$ for $R_{V}=3.1$. Thus, $A_{\mathrm{ACS}, \mathrm{F} 475 \mathrm{~W}} / A_{\mathrm{ACS}, 814 \mathrm{~W}} \sim 2.366$, i.e., $\alpha_{\text {red }} \sim 0.732$ and $A_{\mathrm{WFC} 3, \mathrm{~F} 110 \mathrm{~W}} / A_{\mathrm{ACS}, 814 \mathrm{~W}} \sim 0.5773$. Therefore, combining eqs. 2 and 3 gives an $I$-band extinction value of $A_{I} \sim 0.080 \mathrm{mag}$ for the regions, near the galaxy centre, immediately outside the ACS and WFC3 masked regions (Fig. 2) with a $B-I$ colour of $\sim 2.37$ mag. This $A_{I}$ value corresponds to a low IR extinction value of $A_{J} \sim 0.047 \mathrm{mag}$. For comparison, $A_{I} \sim 0.053 \mathrm{mag}$ for the outer part of the dust disc (as traced by the colour map) near the galaxy centre with a $B-I$ colour of $\sim 2.33$ mag. For the reddest part of the dust disc with $B-I \sim 2.85$ mag, which we have masked, $A_{I} \sim 0.053 \mathrm{mag}$ (see Figs. 1, 2 and 3). Thus, we find a low IR extinction value of $A_{J} \sim 0.031$ mag for the outer part of the dust disc, while for the reddest part $A_{J} \sim 0.250$ mag. These three $A_{J}$ values can be compared to our adopted uncertainty on surface brightnesses $\mu_{\mathrm{b}}, \mu_{\mathrm{e}}$ and $\mu_{0}$, i.e., $\sim 0.02 \mathrm{mag} \operatorname{arcsec}^{-2}$.

We did not attempt to derive a dust-corrected $F 110 W$ surface brightness profile for NGC 5322 by creating a dust-corrected WFC3 $F 110 W$ image of the galaxy using eq. 3. Although the foreground screen approximation (eq. 2) enables us to constrain the uncertainties due to the effects of the nuclear dust, the technique is not fully reliable to derive a robust light profile for the galaxy (Nowak et al. 2008; Bender et al. 2015). Both Nowak et al. (2008, Appendix A) and Bender et al. (2015, Section 3.3) have estimated the best $\alpha_{\text {red }}$ values in a subjective manner by iterating $\alpha_{\text {red }}$ to obtain what they considered the best dust-corrected images for their galaxies.

\subsection{Spheroid, inner disc and outer halo formation and the connection to the central radio source}

Here we discuss the formation of NGC 5322, an E3 - 4 giant elliptical in a poor group (Garcia 1993; Madore et al. 2004), considering the implications of our findings; a galaxy spheroid with a stellar mass deficit $M_{\text {def }} \sim(1.1 \pm 0.3) \times 10^{9} M_{\odot}$, small-scale stellar and dust discs, an outer stellar halo and a central radio source (Section 3.5, see also Feretti et al. 1984; Hummel et al. 1984).

\subsubsection{Spheroid property}

In the previous sections, we have discussed that the depleted core in NGC 5322 is not due to the nuclear dust disc crossing the galaxy center, which can create an artificial core or enlarge a pre-existing depleted core. However, it is still worth comparing the properties of the spheroid of NGC 5322 with those of core-Sérsic galaxies to ensure that our core identification is real. Faber et al. (1997) noted the connection between the "core" vs. "power-law" central structural dichotomy and the "boxy" vs. "discy" and "slow rotator" vs. "fast rotator" divides (see also Davies et al. 1983; Carter 1987; Bender 1988; Bender et al. 
1988; Peletier et al. 1990; Jaffe et al. 1994). In agreement with this picture ${ }^{8}$, NGC 5322's core-Sérsic spheroid rotates slowly (e.g., Bender 1988; Rix \& White 1992; Emsellem et al. 2011; Krajnović et al. 2013a,b) and has boxy isophotes (Figs. 2 and 3). The spheroid's Sérsic $(n \sim 4)$ stellar light distribution outside the core, $M_{V} \sim-21.77 \mathrm{mag} \approx 73.4 \%$ of the total galaxy light (i.e., including the stellar halo) and $\sigma \sim 229 \mathrm{~km} \mathrm{~s}^{-1}$, also suggest that the galaxy is a core-Sérsic galaxy. Moreover, there is a tendency for the AGN of core-Sérsic galaxies to be more radio-loud than those of the coreless galaxies (e.g., Capetti \& Balmaverde 2005, 2006; Kormendy et al. 2009; Richings et al. 2011). Indeed, NGC 5322 is classified as a radio-loud galaxy (e.g., Baldi \& Capetti 2009).

\subsubsection{Kinematically decoupled core}

The gas-poor major merger scenario of the spheroid (Section 4.3.1) cannot naturally account for the galaxy's inner stellar disc which counter-rotates rapidly with respect to the slowly rotating spheroid (Bender 1988, see Section 3.4). Galaxies with central regions that are kinematically decoupled from the rest of the galaxy are thought to be common, but their formation mechanisms are still unclear (e.g., Efstathiou et al. 1982; Bender 1988; Franx \& Illingworth 1988; Hernquist \& Barnes 1991; Balcells \& Quinn 1990; Forbes et al. 1995; Carollo et al. 1997; McDermid et al. 2006; Tsatsi et al. 2015). Integrating our Sérsic fit and using $\epsilon \sim 0.4$, we find an $I$-band $M_{I} \sim-19.29$ mag for the counterrotating disc, dominated at all radii by the spheroid (Fig. 4). This disc only makes up $\approx 2.6 \%$ of the total galaxy light and using a mass-to-light ratio $M / L_{I} \sim 2.8$, the same as the spheroid's $M / L_{I}$, gives a stellar disc mass of $M_{* \text {, disc }} \sim(6.4 \pm 1.8) \times 10^{9} M_{\odot}$, somewhat smaller than the stellar disc mass from Bender $\left(1990, M_{* \text {,disc }} \sim 10^{10} M_{\odot}\right)$. The residual structure along the major axis of the galaxy due to the counterrotating stellar disc (Figs. 1 and 3) is in excellent agreement with the galaxy velocity map by Krajnović et al. (2011, their Fig. C4).

Kinematically decoupled core (KDC) formation due to survived cores of accreted gas-poor satellites has been discussed by Kormendy (1984) and Balcells \& Quinn (1990). These mechanisms are, however, inconsistent with this galaxy's inner dust disc and central radio source which are a telltale signature of nuclear cold gas accretion events. It is unlikely that the KDC of NGC 5322 (i.e., the inner stellar disc) was built through major mergers of gas-rich or partially-gaseous galaxies (e.g., Barnes \& Hernquist 1991; Hernquist \& Barnes 1991; Bender \& Surma 1992; McDermid et al. 2006; Bois et al. 2011; Krajnović et al. 2011; Naab et al. 2014; Toloba et al. 2014; Krajnović et al. 2015; Tsatsi et al. 2015). These processes drive nuclear inflows of gas and subsequently trigger intense central starbursts, which are typically $\sim 1 \%-5 \%$ of the host galaxy light (Hopkins et al. 2008, 2009, see also Park, Smith, \& Yi 2017), resulting in a coreless galaxy (e.g., Faber et al. 1997). Furthermore, using numerical simulations, Hau \& Thomson (1994) proposed a formation model for KDC galaxies, including NGC 5322, where a retrograde flyby interaction of a galaxy with another elliptical galaxy leads to a slowly rotating halo and a rapidly rotating KDC core. Their simulations assume an inner core plus an other halo components for the galaxies, but NGC 5322 has an inner stellar disc, a spheroid, and an outer halo (See Fig. 4 and Section 3).

The properties of NGC 5322 favour the formation of the KDC due to the accretion of a gas-rich satellite which settles at the galaxy centre.

\subsubsection{A three-phase assembly for NGC 5322}

We argue that a more likely scenario for NGC 5322 is a three-phase assembly, in which, the galaxy had its spheroid built earlier through $(\mathcal{N} \sim 2-7)$ violent dry major mergers involving SMBHs (e.g., Faber et al. 1997; Kormendy 1999; Rest et al. 2001; Ravindranath et al. 2001; Trujillo et al. 2004; Lauer et al. 2005, 2007; Bell et al. 2006; Ferrarese et al. 2006 ; Xu et al. 2012; Man et al. 2012; Man, Zirm, \& Toft 2016), followed by the subsequent accretion of (a satellite with) metal-rich gaseous material which settles at the galaxy centre into a rapidly rotating (discy) cold gas reservoir. This, in turn, later fuels the central radio source and converts into the inner counter-rotating stellar disc (see Bertola et al. 1988; Franx \& Illingworth 1988; Faber et al. 1997; Caon et al. 2000; Cappellari et al. 2002). Rix \& White (1992) argued that this stellar disc is embedded in the dynamically hot spheroid (i.e., $\left.V_{\text {rot }} / \sigma \sim 1.4\right)$. The outer halo $(\approx 24.0 \%$ of the total galaxy light) could naturally have grown later via dry minor mergers and the accumulation of stars stripped during flybys (e.g., Zolotov et al. 2009 ; Hilz et al. 2012, 2013; Hirschmann et al. 2015; Rodriguez-Gomez et al. 2016, their Fig. 4). Due to NGC 5322's group environment and therefore the lack of intracluster light (Garcia 1993; Madore et al. 2004), this galaxy's halo and spheroid contribute equally in the outermost regions, compared to BCG halos which typically dominate their host spheroids' light at large radii (e.g., Seigar et al. 2007; Donzelli, Muriel, \& Madrid 2011; Dullo, Graham, \& Knapen 2017).

\footnotetext{
8 A caveat here is that the "core" (Lauer et al. 1995; Faber et al. 1997) and "core-Sérsic" (Graham et al. 2003) structural classifications are not identical. Unlike "core-Sérsic" galaxies, "core" galaxies identified by the Nuker model (e.g., Lauer et al. 1995) do not necessarily contain partially-depleted cores relative to their spheroids' outer Sérsic profile (Dullo \& Graham 2012, 2013, 2014, 2015).
} 


\subsubsection{Low-luminosity AGN feedback}

A key challenge to our three-phase formation picture is that the new star formation due to the gas/gas-rich satellite accretion can refill the depleted core (see Faber et al. 1997). However, the core can be protected if the non-thermal AGN feedback swiftly quenches the in-situ star formation after the stellar disc is formed. Serra et al. (2012) detected neutral hydrogen absorption in NGC 5322, although Hibbard \& Sansom (2003) did not detect neutral hydrogen associated with the galaxy. Using e-MERLIN observations, we tentatively detected neutral hydrogen absorption near the central region of NGC 5322. Also, Spolaor et al. (2010) found a significant $\alpha$ enhancement at the central regions of the galaxy $([\alpha / \mathrm{Fe}] \sim 0.30)$, suggesting short star-formation timescales (e.g., Matteucci \& Tornambe 1987; Thomas, Greggio, \& Bender 1999; Thomas et al. 2005).

Our radio data analysis suggests that NGC 5322 houses a low-luminosity AGN (LLAGN). As noted in Section 3.5, the $1.5 \mathrm{GHz}$ radio continuum emission of the galaxy has a core radio power and brightness temperature of $P_{\text {core }} \sim 6.55 \times 10^{20} \mathrm{~W}$ $\mathrm{Hz}^{-1}$ and $T_{\mathrm{B}, \text { core }} \sim 4.5 \times 10^{7} \mathrm{~K}$, respectively (Table 2), which agree with those those reported for low-luminosity AGN $\left(T_{\mathrm{B}, \mathrm{LLAGN}} \gtrsim 10^{7}\right.$ K e.g., Nagar et al. 2002; Nagar, Falcke, \& Wilson 2005; Nyland et al. 2013 and $P_{\text {core, LLAGN }} \sim 10^{18}-10^{25}$ W $\mathrm{Hz}^{-1}$, e.g., Ho \& Ulvestad 2001). For NGC 5322's radio jets, we measured a low radio power of $P_{\text {jets }} \sim 7.04 \times 10^{20} \mathrm{~W} \mathrm{~Hz}^{-1}$. Using Chandra X-ray spectra and the surface brightness profile of NGC 5322, Balmaverde, Baldi, \& Capetti (2008) argued that a low accretion rate $\left(\approx 0.75 M \odot \mathrm{yr}^{-1}\right)$ of hot gas onto the AGN at the bondi radius of 15 pc is enough to account for these radio jets kinetic power. Fig. 3 reveals that the residual image obtained by subtracting the ELLIPSE model image of the galaxy exhibits two bright cones, tracing the orientations of the radio jets. However, since the jets have a low radio power, these bright cones are less likely to form due to radio mode AGN photoionisation of the interstellar medium of the galaxy (e.g., Croton et al. 2006; Heinz et al. 2006; McNamara et al. 2009).

Furthermore, the modelling of the HST brightness profile shows that NGC 5322 has a normal size core for the galaxy's $\sigma$ and $M_{V}$, thus it is unlikely that a pre-existing large core in this galaxy was partially replenished by new stars (see Section 4.2 ).

\subsubsection{Three-phase scenario versus galaxy properties and environment}

The three-phase formation scenario reconciles well with the colour, stellar population properties, globular cluster system and the environment of NGC 5322. Spolaor et al. (2010, their Tables C1, C2 and C3) reported a positive age gradient for the galaxy where the age rises from a central value of 7.4 Gyr to an average age of 12.6 Gyr. The central intermediate age (7.4 Gyr) stellar population (see also Proctor \& Sansom 2002) is consistent with a stellar population that is a mixture of relatively young and old stars associated mostly to the stellar disc (i.e., KDC) and spheroid, respectively. NGC 5322's negative radial metallicity gradient of $\sim-0.20$ dex decade ${ }^{-1}$ is such that the galaxy is metal-rich in the central regions $([\mathrm{Z} / \mathrm{H}] \sim 0.45]$ and it becomes less metal-rich with increasing radius (Spolaor et al. 2010). This negative metallicity gradient is expected since the small (gas-poor) satellites forming the outer halo are metal-poor. The higher central metallicity may reflect an early dissipative merger-induced starburst in the (coreless) progenitors of the spheroid and may typically remain intact during dry mergers (e.g., De Lucia et al. 2006; Spolaor et al. 2010) and the accretion of (a satellite with) already enriched gaseous material that built up the stellar disc. The galaxy becomes gradually bluer towards larger radii (see Section 3.1), as also reflected in the decline of the fraction of red globular clusters going from the centre to larger radii (Harris et al. 2006, their Table 5 and Fig. 24). In line with the three-phase picture, NGC 5322 resides in a poor galaxy group environment, which favours mergers (e.g., Zabludoff \& Mulchaey 1998; Carlberg et al. 2001).

The nuclear dust disc and the inner stellar disc of NGC 5322 are both oriented along the major-axis of the galaxy, suggesting a common (external) origin for both these structures. The dust disc is nearly perpendicular to the radio jets (Section 3.5). However, Verdoes Kleijn \& de Zeeuw (2005) did not find a trend between the radio jets and dust orientations for their sample of galaxies with nuclear dust disc, in contrast they found that radio jets tend to be perpendicular to the dust structure in the 'dust lane' galaxies. A number of past studies found a strong link between the presence of dust in galaxies and radio activities (e.g., Jaffe et al. 1994; van Dokkum \& Franx 1995; Verdoes Kleijn et al. 1999; Martel et al. 1999; Tran et al. 2001; Verdoes Kleijn \& de Zeeuw 2005; Lauer et al. 2005; Baldi \& Capetti 2008; Shabala et al. 2012). The dust disc in NGC 5322 may signal the accretion of externally acquired gaseous material onto the the central supermassive black hole which feeds the AGN and powers the radio jets, extending $\sim 1.6 \mathrm{kpc}$ at a position angle of $172^{\circ}$.

\section{CONCLUSIONS}

As part of the LeMMINGs survey, we investigated the nuclear activity and central structure of the radio galaxy NGC 5322 using a new sub-arcsec L-band (1.25 - $1.75 \mathrm{GHz})$ e-MERLIN radio observation together with HST WFC3/IR, ACS/WFC and SDSS imaging. We extracted a composite (HST WFC3/IR and ACS/WFC + SDSS) major-axis surface brightness profile and isophotal parameters. The inner $\left(R \lesssim 3^{\prime \prime}\right)$ part of this composite light profile was extracted from the redder HST WFC3/IR image, applying a pixel mask which mapped the galaxy's nuclear dust disc with a radius of $1^{\prime \prime} .7 \approx 246.5$ pc and a thickness of $00^{\prime \prime} 6 \approx 87$ pc near the galaxy center in the $F 814 W /$ ACS/WFC image. For the first time we have decomposed NGC 5322 's 
composite light profile, covering $R \sim 200^{\prime \prime}$, into an inner Sérsic $(n \sim 1 \pm 0.2)$ stellar disc, a boxy core-Sérsic spheroid plus an outer, exponential stellar halo. Our primary findings are follows:

(1) We have derived a central stellar mass deficit $\left(M_{\text {def }} \sim 1.1 \times 10^{9} M_{\odot}\right)$ in the core-Sérsic spheroid with a core size $R_{\mathrm{b}}$ $\sim 0.37 \pm 0 . \prime 04 \approx 53.7 \pm 5.4 \mathrm{pc}$ and $n \sim 4 \pm 0.8$. This core size is in good agreement with those estimated utilising the spheroid's $\sigma \sim 229 \mathrm{~km} \mathrm{~s}^{-1}$ and $M_{V} \sim-21.77 \mathrm{mag}\left(\approx 73.4 \%\right.$ of the total galaxy light) along with the $R_{\mathrm{b}}-\sigma$ and $R_{\mathrm{b}}-M_{V}$ relations for core-Sérsic galaxies (Dullo \& Graham 2014), i.e., $R_{\mathrm{b}, \sigma-\text { based }} \approx 44.0 \pm 6.1 \mathrm{pc}$ and $R_{\mathrm{b}, M_{V} \text {-based }} \approx 48.6 \pm 7.2 \mathrm{pc}$. We also measured low $F 110 W$-band extinction $\left(A_{F 110 W} \sim 0.031-0.047 \mathrm{mag}\right)$ in the regions immediately surrounding the masked regions near the galaxy centre, compared to our adopted uncertainty on the surface brightnesses $\mu_{\mathrm{b}}, \mu_{\mathrm{e}, \mathrm{S}}$ and $\mu_{0, \mathrm{~h}}$, i.e., $\sim 0.02 \mathrm{mag} \operatorname{arcsec}^{-2}$. The good agreement in $R_{\mathrm{b}}$ and the low IR extinction give us confidence that our measurement of NGC 5322's $R_{\mathrm{b}}$ (and therefore $M_{\mathrm{def}}$ ) is not plagued by the effects of the nuclear dust disc. We found a stellar mass $M_{*} \sim(6.4 \pm 1.8) \times 10^{9} M_{\odot}$ for the stellar disc, somewhat smaller than that reported in the past.

(2) We find that NGC 5322 has a core $1.5 \mathrm{GHz}$ radio continuum emission with a power of $P_{\text {core }} \sim 6.55 \times 10^{20} \mathrm{~W} \mathrm{~Hz}^{-1}$ and a brightness temperature of $T_{\mathrm{B}, \text { core }} \sim 4.5 \times 10^{7} \mathrm{~K}$ (Table 2). Both these figures favour a non-thermal nuclear radio emission due to a low-luminosity AGN. For the galaxy's radio jets, we measured a low radio power of $P_{\text {jets }} \sim 7.04 \times 10^{20} \mathrm{~W} \mathrm{~Hz}^{-1}$.

(3) We propose a three-phase formation scenario to explain the formation and evolution of NGC 5322. The spheroid may have built up early through $(2-7)$ gas-poor major mergers, as traced by the $M_{\mathrm{def}} / M_{\mathrm{BH}}$ ratio. The galaxy subsequently cannibalised a satellite containing metal-rich gas, with stars and gas settling at the centre and forming the rapidly rotating stellar disc, counterrotating with respect to the spheroid. The outer stellar halo later grew inside-out via minor mergers and accretion of tidal debris. Our formation model for NGC 5322 is consistent with the galaxy's colour, stellar population properties, globular cluster system and environment.

(4) NGC 5322's dust disc oriented along the galaxy's major-axis is nearly perpendicular to the radio jets, which extend $\sim 1.6 \mathrm{kpc}$ at a position angle of $172^{\circ}$. Nuclear dust features may indicate the accretion of externally acquired gaseous material onto the AGN, powering central radio sources. For NGC 5322, this radio mode (low-luminosity) AGN feedback that drives the low power jets might have quenched the late-time star formation in the galaxy after the stellar disc is formed, preventing the refill of the depleted core.

\section{ACKNOWLEDGMENTS}

We thank the referee for their careful reading of the paper and many constructive suggestions that improved the paper. BTD acknowledges support from a Spanish postdoctoral fellowship "Ayudas para la atracción del talento investigador. Modalidad 2: jóvenes investigadores, financiadas por la Comunidad de Madrid" under grant number 2016-T2/TIC-2039. BTD acknowledges financial support from the Spanish Ministry of Economy and Competitiveness (MINECO) under grant number AYA2016-75808-R. BTD \& JHK acknowledge financial support from MINECO under grant number AYA2013-41243-P. JHK acknowledges financial support from the European UnionâẮ́s Horizon 2020 research and innovation programme under Marie SkÅĆodowska-Curie grant agreement No 721463 to the SUNDIAL ITN network, and from the Spanish Ministry of Economy and Competitiveness (MINECO) under grant number AYA2016-76219-P. IMcH thanks the Royal Society for the award of a Royal Society Leverhulme Trust Senior Research Fellowship. RDB and IMcH also acknowledge the support of STFC under grant [ST/M001326/1]. Based on observations made with the NASA/ESA Hubble Space Telescope, and obtained from the Hubble Legacy Archive, which is a collaboration between the Space Telescope Science Institute (STScI/NASA), the Space Telescope European Coordinating Facility (ST-ECF/ESA) and the Canadian Astronomy Data Centre (CADC/NRC/CSA). e-MERLIN is a National Facility operated by the University of Manchester at Jodrell Bank Observatory on behalf of STFC. Funding for the Sloan Digital Sky Survey IV has been provided by the Alfred P. Sloan Foundation, the U.S. Department of Energy Office of Science, and the Participating Institutions. SDSS-IV acknowledges support and resources from the Center for High-Performance Computing at the University of Utah. The SDSS web site is www.sdss.org.

SDSS-IV is managed by the Astrophysical Research Consortium for the Participating Institutions of the SDSS Collaboration including the Brazilian Participation Group, the Carnegie Institution for Science, Carnegie Mellon University, the Chilean Participation Group, the French Participation Group, Harvard-Smithsonian Center for Astrophysics, Instituto de Astrofísica de Canarias, The Johns Hopkins University, Kavli Institute for the Physics and Mathematics of the Universe (IPMU) / University of Tokyo, Lawrence Berkeley National Laboratory, Leibniz Institut für Astrophysik Potsdam (AIP), Max-PlanckInstitut für Astronomie (MPIA Heidelberg), Max-Planck-Institut für Astrophysik (MPA Garching), Max-Planck-Institut für Extraterrestrische Physik (MPE), National Astronomical Observatories of China, New Mexico State University, New York University, University of Notre Dame, Observatário Nacional / MCTI, The Ohio State University, Pennsylvania State University, Shanghai Astronomical Observatory, United Kingdom Participation Group, Universidad Nacional Autónoma de México, University of Arizona, University of Colorado Boulder, University of Oxford, University of Portsmouth, University of Utah, University of Virginia, University of Washington, University of Wisconsin, Vanderbilt University, and Yale University. 


\section{REFERENCES}

Argo M., 2015, arXiv, arXiv:1502.04936

Bahcall, J. N., Kirhakos, S., Schneider, D. P., et al. 1995, ApJ, 452, L91

Balcells, M., \& Quinn, P. J. 1990, ApJ, 361, 381

Baldi R. D., Capetti A., 2008, A\&A, 489, 989

Baldi R. D., Capetti A., 2009, A\&A, 508, 603

Balmaverde B., Baldi R. D., Capetti A., 2008, A\&A, 486, 119

Barnes, J. E., \& Hernquist, L. E. 1991, ApJ, 370, L65

Becker R. H., White R. L., Helfand D. J., 1995, ApJ, 450, 559

Begelman, M. C., Blandford, R. D., \& Rees, M. J. 1980, Nature, 287, 307

Bell E. F., et al., 2006, ApJ, 640, 241

Bender, R. 1988, A\&A, 202, L5

Bender, R. 1990, Dynamics and Interactions of Galaxies, 232

Bender, R. 1996, New Light on Galaxy Evolution, 171, 181

Bender, R., Doebereiner, S., \& Moellenhoff, C. 1988, A\&AS, 74, 385

Bender, R., Kormendy, J., Cornell, M. E., \& Fisher, D. B. 2015, ApJ, 807, 56

Bender, R., \& Surma, P. 1992, A\&A, 258, 250

Bertin, E., \& Arnouts, S. 1996, A\&AS, 117, 393

Bertola, F., Buson, L. M., \& Zeilinger, W. W. 1988, Nature, 335, 705

Beswick R., Argo M. K., Evans R., McHardy I., Williams D. R. A., Westcott J., 2014, evn..conf, 10

Beswick, R., Brinks, E., Perez-Torres, M., et al. 2015, Advancing Astrophysics with the Square Kilometre Array (AASKA14), 70

Blakeslee, J. P., Lucey, J. R., Tonry, J. L., et al. 2002, MNRAS, 330, 443

Bois M., et al., 2011, MNRAS, 416, 1654

Boylan-Kolchin, M., Ma, C.-P., \& Quataert, E. 2004, ApJ, 613, L37

Caon, N., Macchetto, D., \& Pastoriza, M. 2000, ApJS, 127, 39

Capetti A., Balmaverde B., 2005, A\&A, 440, 73

Capetti A., Balmaverde B., 2006, A\&A, 453, 27

Cappellari, M., Verolme, E. K., van der Marel, R. P., et al. 2002, ApJ, 578, 787

Carlberg, R. G. 1986, ApJ, 310, 593

Carlberg, R. G., Yee, H. K. C., Morris, S. L., et al. 2001, ApJ, 563, 736

Carollo, C. M., Franx, M., Illingworth, G. D., \& Forbes, D. A. 1997, ApJ, 481, 710

Carter, D. 1987, ApJ, 312, 514

Cole, S., Aragon-Salamanca, A., Frenk, C. S., Navarro, J. F., \& Zepf, S. E. 1994, MNRAS, 271,781

Condon J. J., 1992, ARA\&A, 30, 575

Condon J. J., Cotton W. D., Greisen E. W., Yin Q. F., Perley R. A., Taylor G. B., Broderick J. J., 1998, AJ, 115, 1693

Côté P., et al., 2006, ApJS, 165, 57

Cox T. J., Jonsson P., Somerville R. S., Primack J. R., Dekel A., 2008, MNRAS, 384,

Crane, P., Stiavelli, M., King, I. R., et al. 1993, AJ, 106, 1371

Croton, D. J., Springel, V., White, S. D. M., et al. 2006, MNRAS, 365, 11

de Vaucouleurs, G. 1948, Annales d'Astrophysique, 11, 247

de Vaucouleurs, G., de Vaucouleurs, A., Corwin, H. G., Jr., et al. 1991, Third Reference Catalogue of Bright Galaxies (Berlin: Springer)

Davies, R. L., Efstathiou, G., Fall, S. M., Illingworth, G., \& Schechter, P. L. 1983, ApJ, 266, 41

De Lucia, G., Springel, V., White, S. D. M., Croton, D., \& Kauffmann, G. 2006, MNRAS, 366,499

Dullo, B. T., \& Graham, A. W. 2012, ApJ, 755, 163

Dullo, B. T., \& Graham, A. W. 2013, ApJ, 768, 36

Dullo, B. T., \& Graham, A. W. 2014, MNRAS, 444, 2700

Dullo, B. T., \& Graham, A. W. 2015, ApJ, 798, 55

Dullo, B. T., Martínez-Lombilla, C., \& Knapen, J. H. 2016, MNRAS, 462, 3800

Dullo B. T., Graham A. W., Knapen J. H., 2017, MNRAS, 471, 2333

Donzelli C. J., Muriel H., Madrid J. P., 2011, ApJS, 195, 15

Ebisuzaki, T., Makino, J., \& Okumura, S. K. 1991, Nature, 354, 212

Efstathiou, G., Ellis, R. S., \& Carter, D. 1982, MNRAS, 201, 975

Emsellem E., et al., 2011, MNRAS, 414, 888

Faber, S. M., Tremaine, S., Ajhar, E. A., et al. 1997, AJ, 114, 1771

Feretti, L., Giovannini, G., Hummel, E., \& Kotanyi, C. G. 1984, A\&A, 137, 362

Ferrarese, L., van den Bosch, F. C., Ford, H. C., Jaffe, W., \& O'Connell, R. W. 1994, AJ, 108,1598

Ferrarese, L., Côté, P., Jordán, A., et al. 2006, ApJS, 164, 334

Forbes, D. A., Franx, M., \& Illingworth, G. D. 1995, AJ, 109, 1988

Ford, H. C., Bartko, F., Bely, P. Y., et al. 1998, Proc. SPIE, 3356, 234

Franx, M., \& Illingworth, G. D. 1988, ApJ, 327, L55

Fukugita, M., Shimasaku, K., \& Ichikawa, T. 1995, PASP, 107, 945

Garcia, A. M. 1993, A\&AS, 100, 47

Goudfrooij P., Hansen L., Jorgensen H. E., Norgaard-Nielsen H. U., de Jong T., van den Hoek L. B., 1994, A\&AS, 104, 179

Graham, A. W. 2004, ApJ, 613, L33

Graham A. W., Ciambur B. C., Savorgnan G. A. D., 2016, ApJ, 831, 132

Graham, A. W., Erwin, P., Trujillo, I., \& Asensio Ramos, A. 2003, AJ, 125, 2951

Graham, A. W., \& Scott, N. 2013, ApJ, 764, 151 
Graham, A. W., \& Spitler, L. R. 2009, MNRAS, 397, 2148

Gualandris, A., \& Merritt, D. 2008, ApJ, 678, 780-797

Harris, W. E., Whitmore, B. C., Karakla, D., et al. 2006, ApJ, 636, 90

Hau, G. K. T., \& Thomson, R. C. 1994, MNRAS, 270, L23

Heinz, S., Brüggen, M., Young, A., \& Levesque, E. 2006, MNRAS, 373, L65

Hernquist, L. 1992, ApJ, 400, 460

Hernquist, L. 1993, ApJ, 409, 548

Hernquist, L., \& Barnes, J. E. 1991, Nature, 354, 210

Hibbard, J. E., \& Sansom, A. E. 2003, AJ, 125, 667

Hilz, M., Naab, T., Ostriker, J. P., et al. 2012, MNRAS, 425, 3119

Hilz, M., Naab, T., \& Ostriker, J. P. 2013, MNRAS, 429, 2924

Hirschmann, M., Naab, T., Ostriker, J. P., et al. 2015, MNRAS, 449, 528

Ho, L. C., Filippenko, A. V., \& Sargent, W. L. W. 1997, ApJS, 112, 315

Ho L. C., Ulvestad J. S., 2001, ApJS, 133, 77

Hopkins, P. F., Hernquist, L., Cox, T. J., Dutta, S. N., \& Rothberg, B. 2008, ApJ, 679, 156-181

Hopkins P. F., Lauer T. R., Cox T. J., Hernquist L., Kormendy J., 2009, ApJS, 181, 486

Hummel, E., van der Hulst, J. M., \& Dickey, J. M. 1984, A\&A, 134, 207

Jaffe, W., Ford, H. C., O'Connell, R. W., van den Bosch, F. C., \& Ferrarese, L. 1994, AJ, 108, 1567

Jedrzejewski, R. I. 1987, MNRAS, 226, 747

Khochfar, S., \& Burkert, A. 2001, ApJ, 561, 517

Knapen, J. H., Shlosman, I., \& Peletier, R. F. 2000, ApJ, 529, 93

Kobayashi, C., \& Arimoto, N. 1999, ApJ, 527, 573

Kormendy, J. 1984, ApJ, 287, 577

Kormendy, J., Dressler, A., Byun, Y. I., et al. 1994, European Southern Observatory Conference and Workshop Proceedings, 49,147

Kormendy J., 1999, ASPC, 182,

Kormendy J., Fisher D. B., Cornell M. E., Bender R., 2009, ApJS, 182, 216

Krajnović D., et al., 2011, MNRAS, 414, 2923

Krajnović, D., Karick, A. M., Davies, R. L., et al. 2013, MNRAS, 433, 2812a

Krajnović, D., Alatalo, K., Blitz, L., et al. 2013, MNRAS, 432, 1768b

Krajnović D., et al., 2015, MNRAS, 452, 2

Kulkarni, G., \& Loeb, A. 2012, MNRAS, 422, 1306

Lauer, T. R., Ajhar, E. A., Byun, Y.-I., et al. 1995, AJ, 110, 2622

Lauer, T. R., Faber, S. M., Gebhardt, K., et al. 2005, AJ, 129, 2138

Lauer T. R., et al., 2007, ApJ, 662, 808

Madore, B. F., Freedman, W. L., \& Bothun, G. D. 2004, ApJ, 607, 810

Man A. W. S., Toft S., Zirm A. W., Wuyts S., van der Wel A., 2012, ApJ, 744, 85

Man A. W. S., Zirm A. W., Toft S., 2016, ApJ, 830, 89

Martel, A. R., Baum, S. A., Sparks, W. B., et al. 1999, ApJS, 122, 81

Matteucci F., Tornambe A., 1987, A\&A, 185, 51

McDermid, R. M., Emsellem, E., Shapiro, K. L., et al. 2006, MNRAS, 373, 906

McNamara, B. R., Kazemzadeh, F., Rafferty, D. A., et al. 2009, ApJ, 698, 594

Merritt, D. 2006, ApJ, 648, 976

Merritt, D., Milosavljević, M., Favata, M., Hughes, S. A., \& Holz, D. E. 2004, ApJ, 607, L9

Mihos, J. C., \& Hernquist, L. 1994, ApJ, 431, L9

Mundy C. J., Conselice C. J., Duncan K. J., Almaini O., Häußler B., Hartley W. G., 2017, MNRAS, 470, 3507

Naab T., et al., 2014, MNRAS, 444, 3357

Naab T., Khochfar S., Burkert A., 2006, ApJ, 636, L81

Nagar N. M., Falcke H., Wilson A. S., Ulvestad J. S., 2002, A\&A, 392, 53

Nagar N. M., Falcke H., Wilson A. S., 2005, A\&A, 435, 521

Nowak N., Saglia R. P., Thomas J., Bender R., Davies R. I., Gebhardt K., 2008, MNRAS, 391, 1629

Nyland K., et al., 2013, ApJ, 779, 173

Park J., Smith R., Yi S. K., 2017, ApJ, 845, 128

Paturel, G., Petit, C., Prugniel, P., et al. 2003, A\&A, 412, 45

Peletier, R. F., Davies, R. L., Illingworth, G. D., Davis, L. E., \& Cawson, M. 1990, AJ, 100, 1091

Proctor, R. N., \& Sansom, A. E. 2002, MNRAS, 333, 517

Ravindranath, S., Ho, L. C., Peng, C. Y., Filippenko, A. V., \& Sargent, W. L. W. 2001, AJ, 122, 653

Rest, A., van den Bosch, F. C., Jaffe, W., et al. 2001, AJ, 121, 2431

Richings, A. J., Uttley, P., \& Körding, E. 2011, MNRAS, 415, 2158

Rix, H.-W., \& White, S. D. M. 1992, MNRAS, 254, 389

Rodriguez-Gomez, V., Sales, L. V., Genel, S., et al. 2016, arXiv:1609.09498

Rusli, S. P., Erwin, P., Saglia, R. P., et al. 2013, AJ, 146, 160

Savorgnan G. A. D., Graham A. W., 2016, ApJS, 222, 10

Schlafly E. F., Finkbeiner D. P., 2011, ApJ, 737, 103

Schweizer, F., Seitzer, P., Faber, S. M., et al. 1990, ApJ, 364, L33

Schweizer, F., \& Seitzer, P. 1992, AJ, 104, 1039

Scorza, C., \& Bender, R. 1995, A\&A, 293, 20

Seigar, M. S., Graham, A. W., \& Jerjen, H. 2007, MNRAS, 378, 1575

Serra P., et al., 2012, MNRAS, 422, 1835 
Shabala S. S., et al., 2012, MNRAS, 423, 59

Silva, D. R., \& Bothun, G. D. 1998, AJ, 116, 2793

Somerville R. S., Davé R., 2015, ARA\&A, 53, 51

Spolaor, M., Kobayashi, C., Forbes, D. A., Couch, W. J., \& Hau, G. K. T. 2010, MNRAS, 408, 272

Springel V., et al., 2005, Natur, 435, 629

Surace, J. A., Sanders, D. B., \& Evans, A. S. 2001, AJ, 122, 2791

Thomas D., Greggio L., Bender R., 1999, MNRAS, 302, 537

Thomas D., Maraston C., Bender R., Mendes de Oliveira C., 2005, ApJ, 621, 673

Toloba E., et al., 2014, ApJ, 783, 120

Tomita A., Aoki K., Watanabe M., Takata T., Ichikawa S.-i., 2000, AJ, 120, 123

Tonry, J. L., Dressler, A., Blakeslee, J. P., et al. 2001, ApJ, 546, 681

Tsatsi, A., Macciò, A. V., van de Ven, G., \& Moster, B. P. 2015, ApJ, 802, L3

Tran, H. D., Tsvetanov, Z., Ford, H. C., et al. 2001, AJ, 121, 2928

Trujillo, I., Erwin, P., Asensio Ramos, A., \& Graham, A. W. 2004, AJ, 127, 1917

Turner M. L., Côté P., Ferrarese L., Jordán A., Blakeslee J. P., Mei S., Peng E. W., West M. J., 2012, ApJS, 203, 5

van Dokkum, P. G., \& Franx, M. 1995, AJ, 110, 2027

Verdoes Kleijn, G. A., Baum, S. A., de Zeeuw, P. T., \& O’Dea, C. P. 1999, AJ, 118, 2592

Verdoes Kleijn, G. A., \& de Zeeuw, P. T. 2005, A\&A, 435, 43

Williams D. R. A., et al., 2017, MNRAS, 472, 3842

Werner N., et al., 2014, MNRAS, 439, 2291

Westcott, J., Brinks, E., Beswick, R. J., et al. 2017, MNRAS, 467, 2113

White, S. D. M., \& Rees, M. J. 1978, MNRAS, 183, 341

Worthey, G. 1994, ApJS, 95, 107

Xu C. K., Zhao Y., Scoville N., Capak P., Drory N., Gao Y., 2012, ApJ, 747, 85

Zabludoff, A. I., \& Mulchaey, J. S. 1998, ApJ, 496, 39

Zolotov, A., Willman, B., Brooks, A. M., et al. 2009, ApJ, 702, 1058 